\title{
Extensive variation between tissues in allele specific expression in an outbred mammal
}

Amanda J. Chamberlain ${ }^{1,2+}$, Christy J. Vander Jagt ${ }^{1,2+}$, Benjamin J. Hayes ${ }^{1,2,3^{*}}$, Majid Khansefid ${ }^{1,2,5}$, Leah C. Marett ${ }^{4}$, Catriona A. Millen ${ }^{2,5}$, Thuy T. T. Nguyen ${ }^{1}$ and Michael E. Goddard ${ }^{1,5}$

\begin{abstract}
Background: Allele specific gene expression (ASE), with the paternal allele more expressed than the maternal allele or vice versa, appears to be a common phenomenon in humans and mice. In other species the extent of ASE is unknown, and even in humans and mice there are several outstanding questions. These include; to what extent is ASE tissue specific? how often does the direction of allele expression imbalance reverse between tissues? how often is only one of the two alleles expressed? is there a genome wide bias towards expression of the paternal or maternal allele; and finally do genes that are nearby on a chromosome share the same direction of ASE? Here we use gene expression data (RNASeq) from 18 tissues from a single cow to investigate each of these questions in turn, and then validate some of these findings in two tissues from 20 cows.
\end{abstract}

Results: Between 40 and 100 million sequence reads were generated per tissue across three replicate samples for each of the eighteen tissues from the single cow (the discovery dataset). A bovine gene expression atlas was created (the first from RNASeq data), and differentially expressed genes in each tissue were identified. To analyse ASE, we had access to unambiguously phased genotypes for all heterozygous variants in the cow's whole genome sequence, where these variants were homozygous in the whole genome sequence of her sire, and as a result we were able to map reads to parental genomes, to determine SNP and genes showing ASE in each tissue. In total 25,251 heterozygous SNP within 7985 genes were tested for ASE in at least one tissue. ASE was pervasive, $89 \%$ of genes tested had significant ASE in at least one tissue. This large proportion of genes displaying ASE was confirmed in the two tissues in a validation dataset.

For individual tissues the proportion of genes showing significant ASE varied from as low as 8-16\% of those tested in thymus to as high as $71-82 \%$ of those tested in lung. There were a number of cases where the direction of allele expression imbalance reversed between tissues. For example the gene SPTY2D1 showed almost complete paternal allele expression in kidney and thymus, and almost complete maternal allele expression in the brain caudal lobe and brain cerebellum. Mono allelic expression (MAE) was common, with 1349 of 4856 genes (28\%) tested with more than one heterozygous SNP showing MAE. Across all tissues, $54.17 \%$ of all genes with ASE favoured the paternal allele. Genes that are closely linked on the chromosome were more likely to show higher expression of the same allele (paternal or maternal) than expected by chance. We identified several long runs of neighbouring genes that showed either paternal or maternal ASE, one example was five adjacent genes (GIMAP8, GIMAP7 copy1, GIMAP4, GIMAP7 copy 2 and GIMAP5) that showed almost exclusive paternal expression in brain caudal lobe.

\footnotetext{
* Correspondence: ben.hayes@ecodev.vic.gov.au

${ }^{\dagger}$ Equal contributors

'Department of Economic Development, Jobs, Transport and Resources,

Agribiosciences Building, 5 Ring Rd, Bundoora, Australia

${ }^{2}$ Dairy Futures Cooperative Research Centre, Agribiosciences Building, 5 Ring

Rd, Bundoora, Australia

Full list of author information is available at the end of the article
}

\section{Biomed Central}

(c) 2015 Chamberlain et al. Open Access This article is distributed under the terms of the Creative Commons Attribution 4.0 International License (http://creativecommons.org/licenses/by/4.0/), which permits unrestricted use, distribution, and reproduction in any medium, provided you give appropriate credit to the original author(s) and the source, provide a link to the Creative Commons license, and indicate if changes were made. The Creative Commons Public Domain Dedication waiver (http://creativecommons.org/publicdomain/zero/1.0/) applies to the data made available in this article, unless otherwise stated. 
(Continued from previous page)

Conclusions: Investigating the extent of ASE across 18 bovine tissues in one cow and two tissues in 20 cows demonstrated 1) ASE is pervasive in cattle, 2) the ASE is often MAE but ranges from MAE to slight overexpression of the major allele, 3) the ASE is most often tissue specific and that more than half the time displays divergent allele specific expression patterns across tissues, 4) across all genes there is a slight bias towards expression of the paternal allele and 5) genes expressing the same parental allele are clustered together more than expected by chance, and there are several runs of large numbers of genes expressing the same parental allele.

Keywords: Gene expression, Differential expression, Tissue specific expression, Allele specific expression, Bovine, Cattle, Transcriptomics, RNA sequencing, Regulation

\section{Background}

There is increasing evidence that many, if not the majority, of mutations that give rise to variation in complex traits reside in regulatory elements that alter gene expression (reviewed by [1]). Mutations in putative regulatory regions have been associated with $>100$ phenotypes in human and other species [2,3] including the classic blond hair phenotype found in northern Europeans ([4], as a result of a variant in the regulatory enhancer of KIT ligand) and stature in cattle ([5], a result of variants in the promoter of PLAG1).

Mutations that affect the expression of an allele on the same chromosome are known as cis expression quantitative trait loci (cis eQTL). If an individual is heterozygous for such a mutation it is expected that the two alleles of the gene will be expressed unequally causing allelic imbalance or allele specific expression (ASE). This ASE can be detected using RNA sequencing (RNAseq) provided there is a heterozygous site in the coding sequence of the gene. ASE can also be caused by imprinting where an epigenetic mark distinguishes the paternal and maternal chromosomes and causes them to be expressed unequally. Evidence is accumulating from studies in mice and humans that regulatory variation that affects the level of expression, observed as allele specific expression or allelic imbalance, is extremely common. For example Crowley et al. [6] reported that greater than $80 \%$ of mouse genes have cis regulatory variation, and estimates from humans and mice range from 4 to $89 \%$ for the proportion of genes showing ASE in at least one tissue (Additional file 1: Table S1).

ASE has been evaluated in a number of studies in humans and mouse, the most extensive being that most recently published by the GTEx consortium [7] in humans. The GTEx consortium used RNAseq data from 29 solid organ tissues, 11 brain sub-regions, whole blood, lymphoblastoid cell lines (LCL) and skin fibroblast cells to study gene expression, including ASE within tissue. They tested for ASE in SNP that were both heterozygous and had greater than 30 RNAseq reads, however they only did this within sample, and found that $1.5-3.7 \%$ of SNP tested showed ASE. Investigating the extent and patterns of ASE in species other than humans and mice, would give insights into the evolution of regulatory variation. Here we report on the extent and pattern of ASE across 18 tissues in a single cow.

Even within humans and mice, let alone other species, there are a number of questions concerning ASE that have only partial answers to date. For instance, to what extent is ASE tissue specific; how often does the direction of imbalance reverse between tissues; how often is only one of the two alleles expressed (mono-allelic expression or MAE); is there a bias towards expression of the paternal or maternal allele; and do genes that are nearby on a chromosome share the same direction of ASE? As we have profiled ASE across so many tissues, our results provide new insights into these questions. As observed for human and mouse, we found pervasive ASE. The majority of ASE was tissue specific. And we also found that more than half of all genes show divergent allele specific patterns across tissues, expressing the paternal allele more highly in one tissue and the maternal allele more highly in another tissue. Further, we found many cases of runs of consecutive genes on a chromosome that were expressed from the same chromosome (paternal or maternal) in a tissue specific manner. Our design does not allow us to distinguish ASE due to cis eQTL from that due to imprinting but we report ASE results separately for a group of genes known to be imprinted in cattle, mouse and human and find a complex pattern showing a degree of ASE that varies between tissues.

In order to assess the extent of ASE within and across tissues in a novel outbred species, namely cattle, we collected 18 tissues at a single point in time from one lactating cow. This paper presents results of differences in gene expression between these tissues - the first bovine gene expression atlas using RNASeq data. We then present the discovery of genes showing ASE and present the extent and relationship of this ASE across tissues. ASE was assessed by mapping to parental genomes of the cow sampled, allowing us to minimise any reference bias [8]. We then validate some of these findings in a dataset consisting of two tissues sampled from 20 lactating cows. 


\section{Results and discussion}

Between six and 100 million paired sequence reads were generated per library, with three replicate samples for each of the eighteen tissues investigated (Additional file 1: Table S2), resulting in between 47 and 176 million paired sequence reads per tissue. On average $92 \%$ of reads aligned to the reference genome for each library, with greater than $70 \%$ mapping uniquely (Additional file 1: Table S2). Reads mapping to ribosomal RNA (rRNA) genes accounted for less than $0.0001 \%$ of total reads for each sample indicating minimal rRNA contamination during library preparation. Mean coverage of expressed transcripts (5' to 3'), showed coverage biased toward the 3' end of transcripts. This is illustrated in Additional file 1: Figure S1 and formally confirmed by a significant regression showing that more reads came from the later exons within a gene (as will be discussed later). This bias is likely a result of some degraded mRNA being sequenced but is small and unlikely to affect subsequent results.

\section{Tissue specific expression (TSE)}

RNAseq reads from each library were aligned to the Ensemble annotation of UMD3.1 bovine reference genome. The total number of expressed genes in each tissue were similar (average 16,935), with mammary gland having the lowest $(15,437)$ and thymus the highest $(18,795)$ number of expressed genes. Within a tissue, the number of reads per gene followed a log-normal distribution (Additional file 1: Figure S2), with many genes contributing close to the average number of reads $\left(10^{-5}\right.$ of all reads) but a few genes contributing 10 times more or 10 times fewer than the average number of reads. As expected transcripts from mammary gland were dominated by the major milk proteins, with $62 \%$ of transcripts from alpha S1 (CSN1S1), alpha S2 (CSN1S2), beta (CSN2) or kappa (CSN3) casein genes, reducing the number of genes observed with low expression. On the other hand thymus was a tissue with high coverage (Additional file 1: Table S2), and this could mean that it had more low abundant transcripts sequenced accounting for the large number of genes expressed.

A tissue by gene counts matrix was produced and used to perform differential expression (DE) analysis in which genes that were more expressed in a tissue than in all other tissues (over-expressed), or less often expressed than in other tissues (under-expressed) were identified. With the exception of blood and leg muscle, all other tissues had a greater number of up-regulated genes than down-regulated (Fig. 1). A full list of the genes DE per tissue is contained in Additional file 2: Table S3. When tissues were clustered on their gene expression patterns, using the full normalised count matrix for all genes, tissues were largely grouped together into clusters reflecting their biological relationship, for example muscle tissues clustered together, brain tissues clustered together and skin tissues clustered together (Fig. 2). Liver and blood were both different from the rest of the tissues and each other, as indicated by the height of the dendrogram branches.

Functional annotation of the DE genes in each tissue (Additional file 2: Table S4 and S5) identified many of the biological processes and pathways already reported as having an established role in those particular tissues. For example, in black skin many of the genes such as Keratins, Keratin associated proteins and Dermal allergen genes have known roles in the skin and this was reflected in the enrichment of Gene Ontology (GO) terms for the biological processes 'epidermis development,' 'ectoderm development', 'hair follicle development' and 'keratinization'. The Kyoto Encyclopaedia of Genes and Genomes (KEGG) pathway 'melanogenesis', responsible for the deposition of melanin in the skin, is also enriched in the DE genes from black skin. For mammary gland the casein genes, alpha lactalbumin $(L A L B A)$ and lactoperoxidase $(L P O)$ all have well defined roles in the mammary gland. The significantly enriched GO terms 'epithelium development', 'epithelial cell differentiation', 'tissue development' and the KEGG pathway 'tight junction', all reflect the mammary gland epithelial cell and tight junction formation, all vital for milk secretion. The GO term 'defence response to bacterium' is a term that reflects the need for mammary tissue to constantly fight bacterial infections which can occur through the teat canal.

Counts were also made on an exon basis and this enabled us to ask whether some exons within a gene were more highly expressed than others, and did this vary between tissues? To summarise the sources of variation in transcription the model $y=\mu+$ tissue + replicate + tissue. replicate + exon number tissue + gene + gene. exon + gene. tissue + exon number + error (see Methods) was fitted, where $y=\log ($ read count of each exon within a tissue and replicate), and the variance accounted for by each random effect (gene, gene.tissue, gene.exon and error) in the model was estimated (Additional file 1: Table S6, and solutions for exon $_{\text {number }}$ presented in Additional file 1: Table S7). All fixed effects (tissue, replicate, tissue.replicate,

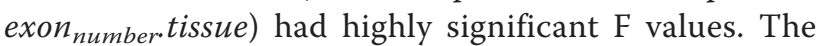
gene.exon effect accounted for the largest amount of variance $(40 \%)$, followed by the gene effect (34\%). The large variance in expression between genes reflects the fact that some genes are highly expressed in all tissues, and others genes are expressed at low levels. The smaller gene.tissue effect $(12 \%$ of total variance) reflects differences between tissues in the genes that are highly expressed. The large gene.exon 


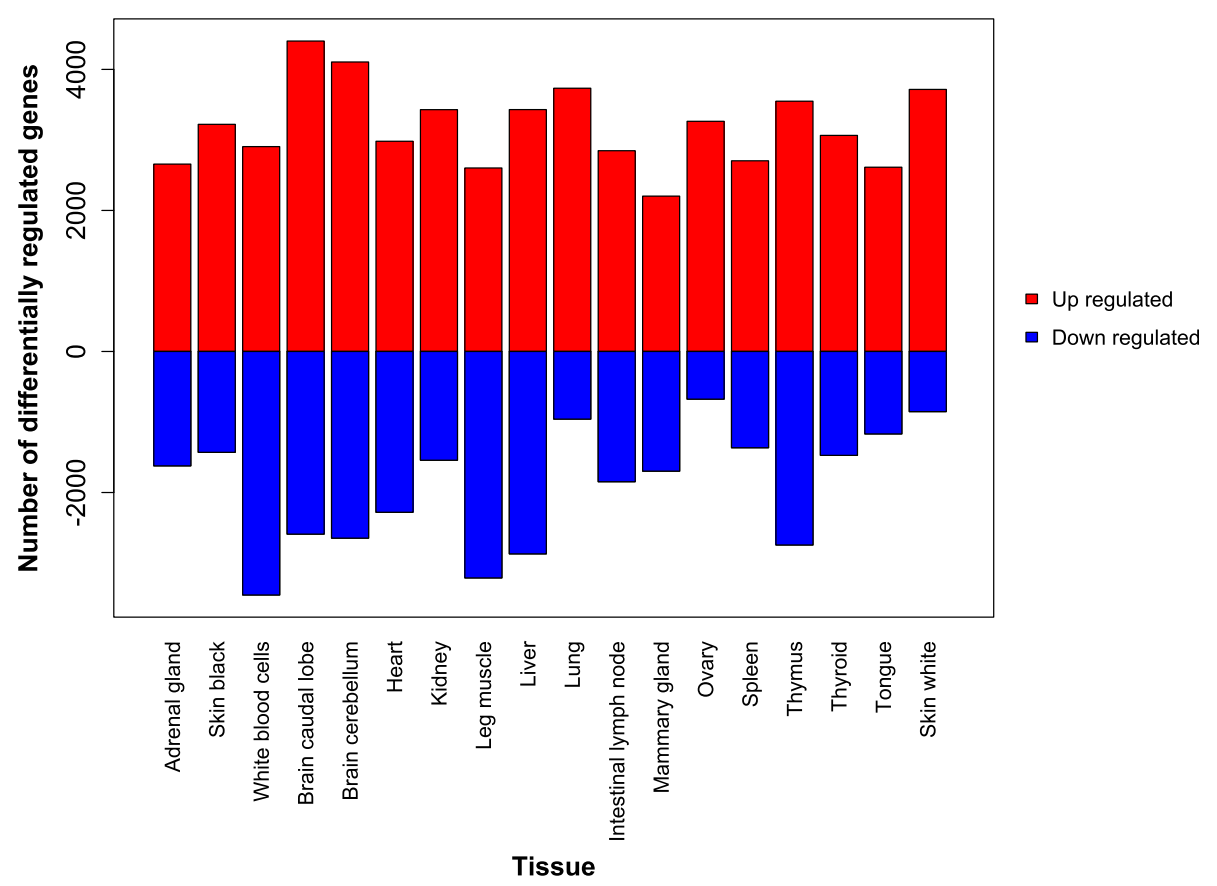

Fig. 1 The number of genes up or down regulated with significant $(p<0.01)$ tissue specific expression (TSE) and a greater than two-fold difference in expression, when compared to the average expression of that gene across all other tissues

effect is due to the fact that some exons within a gene had much higher counts than other exons within the same gene regardless of tissue, even after accounting for any 3' bias in coverage of transcripts (Additional file 1: Figure S1) by fitting exon number (order) in the model. This large gene.exon effect implies there could have been a lot of alternative splicing, or alternatively that the annotation of the bovine genome was poor, so that some exons weren't real or were only occasionally being transcribed. The effect of exon number is due to a bias towards sequencing more exons toward the 3 ' end of the gene. This bias varies between tissues (Additional file 1: Table S7), where adrenal gland was the most affected (the most biased) and therefore had the largest effect and mammary and blood were the least affected (least biased) and had the smallest effect of this bias.

\section{Extent of allele specific expression across tissues}

We had access to unambiguously phased genotypes for all heterozygous variants in the cow's whole genome sequence, where these variants were homozygous in the whole genome sequence of her sire. As a result we were able to map reads to parental genomes [8], to determine SNP and genes showing ASE in the 18 bovine tissues. RNAseq reads from the three replicate libraries were merged and aligned to two parental genomes (see Methods). For all known heterozygous sites that occurred within an exon, allele counts for each alignment were extracted and ASE tested using a Chi-squared test (see Methods) that accounts for any remaining mapping bias. There was very little bias toward the reference allele for all 25,251 SNP tested (Fig. 3). The exception to this was for SNPs that showed mono-allelic expression, it was more often the reference allele than the alternative allele that was expressed (we define MAE as ASE where the frequency of the major allele exceeds 0.9). This could be due to errors in the genome sequence of this cow, with SNP called heterozygous when in fact the cow is homozygous at that position. Such artefacts were also observed by Baran et al. [9] in the 1000 human genome whole genome sequence data and GenotypeTissue Expression (GTEx) RNAseq data. So the following efforts were made to identify and remove sequencing errors, 1) "private" variant detection was undertaken for this cow, and any SNP that were not discovered in that private detection removed, 2) variants only detected in this cow's genome and not in any other animal in the 1000 bull genomes project (Daetwyler et al. [10]) were removed, 3) putative runs of homozygosity $(\mathrm{RoH})$ were identified in whole genome sequence data and heterozygous variants falling within those regions were removed using an approach similar to Macleod et al. [11]. After these quality control steps, there was only a slight bias towards expression of the reference allele (mean frequency 0.5014) and this was eliminated when the MAE classes were removed from the data (Fig. 3, Additional file 1: Table S8). 

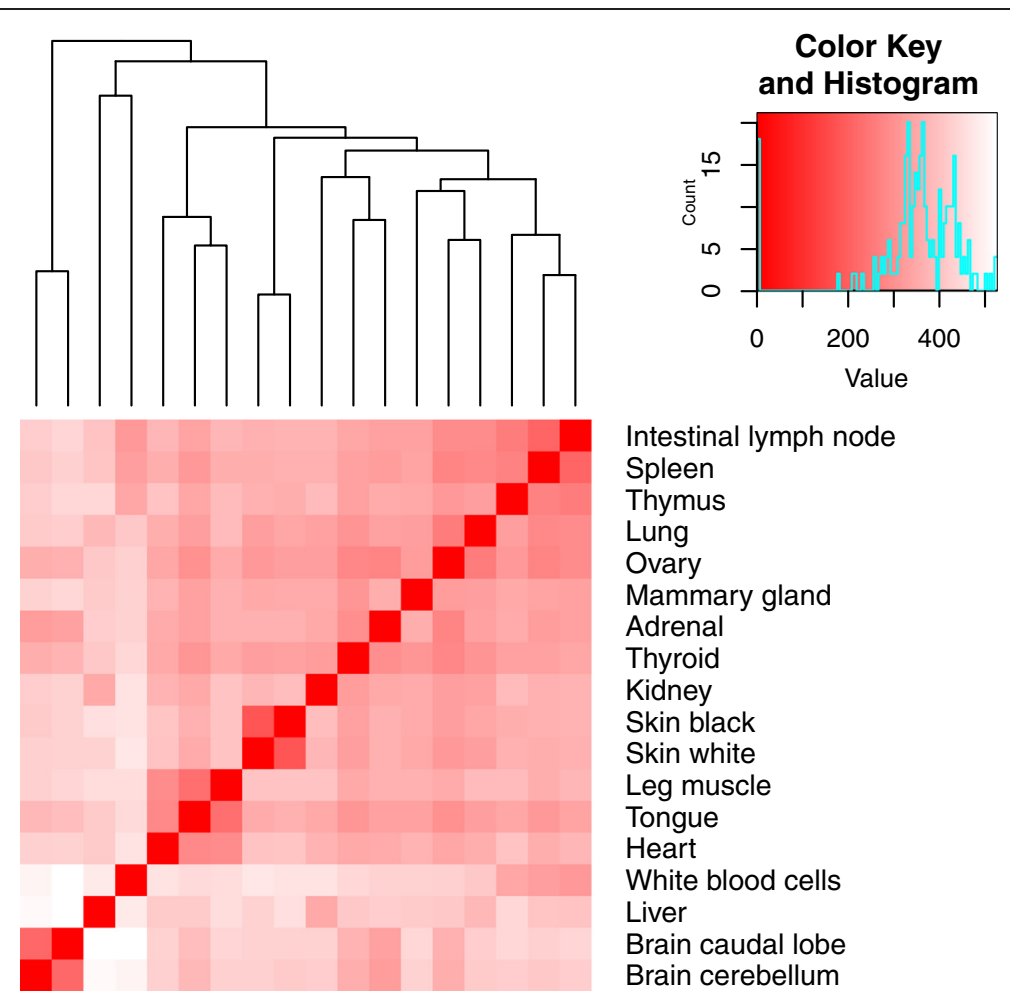

Intestinal lymph node

Spleen

Thymus

Lung

Ovary

Mammary gland

Adrenal

Thyroid

Kidney

Skin black

Skin white

Leg muscle

Tongue

Heart

White blood cells

Liver

Brain caudal lobe

Brain cerebellum

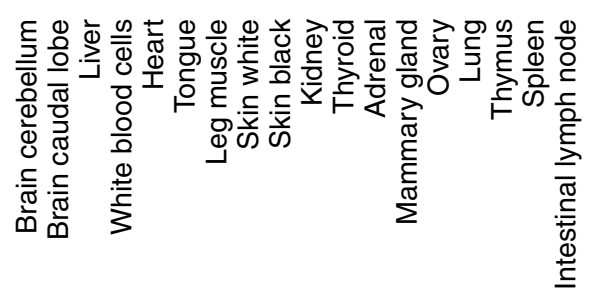

Fig. 2 Tissue x Tissue heat map and hierarchical clustering of gene expression data. Colour key indicates the level of expression "relatedness" between tissue types, the darker the colour, the more similar the pattern of gene expression. The histogram in the colour key represents a density plot of the frequency of distance values. The variability in gene expression between tissues is represented by the height of the dendrogram branches

In total 25,251 heterozygous SNP within 7985 genes were tested for ASE in at least one tissue. $89 \%$ of these 7985 genes tested had at least one SNP with significant ASE, in at least one tissue (Table 1). Wang et al. [12] state that genes with multiple SNP supporting ASE have a higher rate of successful verification. Although, a large proportion $(49 \%)$ of the genes had only 1 SNP within the gene able to be tested, we found ASE for 3570 of 4856 genes with greater than one SNP tested (i.e. $74 \%$ of genes with more than one heterozygous SNP tested). Therefore we estimate that between 74 and $89 \%$ of genes show ASE in at least one tissue and we conclude that ASE is pervasive in cattle. This estimate is higher than the majority of published literature of 4 to $53 \%$ (Additional file 1: Table S1) from whole genome assessment of ASE. The notable exception is the recent publication from Crowley et al. [6] which reported $89 \%$ of all genes tested in mouse brain showed ASE.
Using only those SNP where parental origin could be definitively determined, and where more than 1 SNP was tested within a gene, we found $72 \%$ overall agreement, up to $82 \%$ in thymus, regarding which allele (paternal or maternal) was expressed between SNP in the same gene (Table 2, Fig. 4), and even better agreement where SNP were in the same exon (Table 2). It is worth noting that lung and liver had the lowest concordance between SNP within the same genes and these are the two tissues with the highest proportion of genes showing ASE (Table 1). It is evident from Fig. 4 that where neighbouring SNP within a gene are both significantly ASE both express the same parental allele, and at very similar frequencies. However it also shows that in a few cases ASE switched between maternal and paternal expression within the gene. This is clearly demonstrated for individual genes in detail in Additional file 1: Figure S3A. Clearly here we can see that some genes showed 

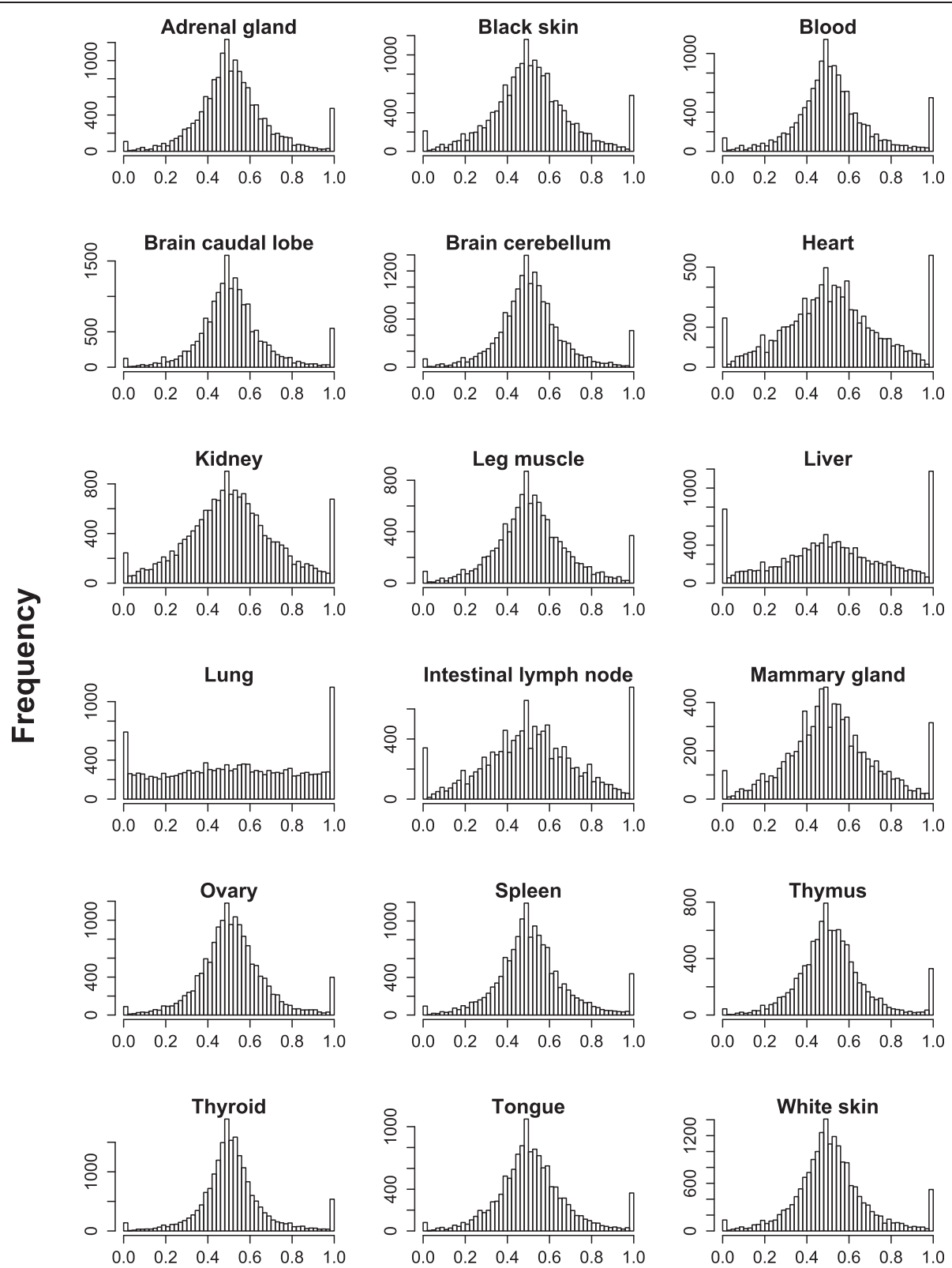

\section{Reference allele frequency}

Fig. 3 Distribution of reference allele frequency after removing SNP that were likely to be a sequencing error, within tissue type, for all SNP tested for ASE. If there was significant mapping bias still present after mapping to the two parental genomes the distribution of allele frequencies would be skewed toward the reference allele. Instead it followed a normal distribution with a peak at reference allele frequency of 0.5 , except for those SNP with reference allele frequency of 0 or 1 . As discussed in the text these represent SNP that display monoallelic expression and even though attempts were made to remove SNP that were due to sequencing errors, higher peaks at reference allele frequency of 1 , as compared with 0 , indicate some still remain

consistent parental allelic expression across the gene, whereas in other cases the expression changes from one parental allele to another within the gene (Additional file 1: Figure S3A). Such patterns of expression have been previously reported by Wood et al. [13] in human brain and liver and are indicative of allele specific isoform expression. As will be discussed later even the same gene showed different patterns of expression across all 18 tissues types (Additional file 1: Figure S3B, C, D and E).

For individual tissues the proportion of genes showing significant ASE varied from as low as $8-16 \%$ of those tested in thymus to as high as $71-82 \%$ of those tested 
Table 1 Allele specific expression analysis results, the number of SNP tested and the number and proportion that showed significant ASE (ASE SNP) in each tissue and in total. Also the number of genes containing SNP tested for ASE (Genes tested) and genes containing greater than one SNP tested for ASE (Genes w/ >1 SNP tested) and then the number and proportion that contained SNP significant for ASE (Genes W/ ASE SNP) and the number and proportion that contained greater than one SNP significant for ASE (Genes w/ >1 ASE SNP) in each tissue and in total. Then finally the number and proportion of genes tested that showed significant ASE in at least one but not all tissues tested (Genes w/ TS ASE SNP) in each tissue and in total

\begin{tabular}{|c|c|c|c|c|c|c|c|}
\hline Tissue & SNP tested & $\begin{array}{l}\text { ASE SNP } \\
\text { (\% tested) }\end{array}$ & Genes tested & $\begin{array}{l}\text { Genes } w />1 \\
\text { SNP tested }\end{array}$ & $\begin{array}{l}\text { Genes w/ ASE } \\
\text { SNP (\% tested) }\end{array}$ & $\begin{array}{l}\text { Genes w/ >1 ASE } \\
\text { SNP (\% tested) }\end{array}$ & $\begin{array}{l}\text { Genes w/ TS ASE } \\
\text { SNP (\% tested) }\end{array}$ \\
\hline Adrenal gland & 14,698 & $2636(18 \%)$ & 5462 & 3134 & 1635 (30 \%) & $536(17 \%)$ & $1502(27 \%)$ \\
\hline Brain caudal lobe & 16,594 & 2419 (15\%) & 5946 & 3483 & $1478(25 \%)$ & 494 (14\%) & 1318 (22 \%) \\
\hline Brain cerebellum & 15,460 & 2324 (15 \%) & 5650 & 3269 & 1470 (26 \%) & $466(14 \%)$ & 1337 (24 \%) \\
\hline Heart & 9545 & 2919 (31 \%) & 3999 & 2118 & 1869 (47 \%) & $618(29 \%)$ & $1768(44 \%)$ \\
\hline Intestinal lymph & 11,719 & 3554 (30 \%) & 4684 & 2542 & 2391 (51 \%) & 782 (31 \%) & 2284 (49 \%) \\
\hline Kidney & 16,616 & 7442 (45 \%) & 5925 & 3457 & 3958 (67 \%) & $1751(51 \%)$ & $3750(63 \%)$ \\
\hline Leg muscle & 11,401 & 2006 (18 \%) & 4455 & 2467 & 1394 (31 \%) & 402 (16 \%) & $1286(29 \%)$ \\
\hline Liver & 12,507 & $6773(54 \%)$ & 4887 & 2715 & 3574 (73 \%) & $1612(59 \%)$ & 3381 (69 \%) \\
\hline Lung & 14,238 & 9216 (65 \%) & 5419 & 3032 & 4448 (82 \%) & 2157 (71 \%) & 4291 (79 \%) \\
\hline Mammary gland & 8161 & 1543 (19 \%) & 3566 & 1838 & 1100 (31\%) & $302(16 \%)$ & $1002(28 \%)$ \\
\hline Ovary & 15,108 & 2043 (14 \%) & 5588 & 3229 & $1407(25 \%)$ & 399 (12\%) & $1264(23 \%)$ \\
\hline Skin black & 16,255 & 4507 (28 \%) & 5870 & 3386 & 2776 (47 \%) & 999 (30 \%) & 2608 (44 \%) \\
\hline Skin white & 17,087 & 3533 (21 \%) & 6004 & 3531 & 2156 (36 \%) & 766 (22\%) & 1997 (33 \%) \\
\hline Spleen & 14,495 & 2066 (14 \%) & 5317 & 3071 & 1448 (27 \%) & $382(12 \%)$ & $1320(25 \%)$ \\
\hline Thymus & 9781 & 986 (10 \%) & 3981 & 2159 & $634(16 \%)$ & $182(8 \%)$ & $501(13 \%)$ \\
\hline Thyroid & 18,181 & 3279 (18 \%) & 6196 & 3703 & 2013 (32 \%) & 688 (19\%) & $1842(30 \%)$ \\
\hline Tongue & 12,744 & 1671 (13 \%) & 4850 & 2718 & 1177 (24 \%) & 327 (12\%) & 1057 (22 \%) \\
\hline White blood cells & 12,768 & 2662 (21 \%) & 4680 & 2690 & $1543(33 \%)$ & $552(21 \%)$ & 1418 (30 \%) \\
\hline Total & 25,251 & 19,082 (76 \%) & 7985 & 4856 & 7067 (89 \%) & $3570(74 \%)$ & $6631(83 \%)$ \\
\hline
\end{tabular}

in lung (Table 1). Pant et al. [14] reported $53 \%$ of genes tested showed significant ASE when testing 1389 genes in human white blood cells. Our estimate of $21-33 \%$ of genes tested in white blood cells was lower but we tested more genes (4680, Table 1). Gao et al. [15] reported $30 \%$ of the 8779 genes tested in human mammary epithelial cell lines showed significant ASE, which agrees well with $16-31 \%$ of the 3566 genes tested in mammary gland in this study. Our results in lung indicate that $71-82 \%$ of 5419 genes tested showed significant ASE in this tissue, the highest of any tissue tested. This is much higher than the $2 \%$ of SNP showing ASE estimated by the GTEx Consortium [7], however we tested many more SNP and the GTEx Consortium tested only SNP that were in common across samples and reported only the within sample estimate of ASE. Our estimate of 14-25 and 14-26\% of the 5462 and 5946 genes tested in brain caudal lobe and brain cerebellum respectively are much lower than the estimate of $89 \%$ by Crowley et al. [6] in whole mouse brain, however they had an extremely powerful design testing ASE in greater than 12,000 genes in 96 individuals from all possible pairwise crosses between the three divergent inbred lines, as opposed to the single outbred individual tested here. The power of our study comes from testing many tissues, as did Gregg et al. [16] who tested 52 tissue samples in mouse, all from the mouse brain, and the GTEx Consortium [7] which tested 43 different tissue samples from 175 humans. The GTEx Consortium estimated $1.5-3.7 \%$ of SNP tested showed ASE, however they only tested an average of 6385 SNP, a lot less than that tested here, and a lot less than Crowley et al. [6], and their estimates are within sample within tissue and not across all sample and tissues as is reported by Crowley et al. and this study.

Of the genes showing significant ASE, $94 \%$ displayed ASE in only a subset of the tissues they were expressed in ("Genes w/ TS ASE SNP" in Table 1). Figure 5a demonstrates that ASE was correlated between tissues, where red indicates ASE was correlated, and white indicates it was not. The tissues are clustered based on their ASE and the variability in ASE between tissues is represented by the height of the dendrogram branches. Tissues with similar function, such as black and white skin, cluster together as they did with gene expression (Fig. 2). However some ASE relationships are different 
Table 2 Using definitively phased SNP this table shows the number of genes and exons with greater than one SNP in them and the number and proportion of those tested where the same parental allele was expressed, i.e. the SNP were concordant

\begin{tabular}{|c|c|c|c|c|}
\hline Tissue & $\begin{array}{l}\text { Genes with >1 } \\
\text { ASE SNP }\end{array}$ & $\begin{array}{l}\text { Genes with concordant } \\
\text { SNP (\% tested) }\end{array}$ & $\begin{array}{l}\text { Exons with }>1 \\
\text { ASE SNP }\end{array}$ & $\begin{array}{l}\text { Exons with concordant } \\
\text { SNP (\% tested) }\end{array}$ \\
\hline Adrenal gland & 347 & $278(80.1 \%)$ & 224 & $194(86.6 \%)$ \\
\hline Brain caudal lobe & 314 & $358(66.7 \%)$ & 196 & $230(74.9 \%)$ \\
\hline Brain cerebellum & 334 & $249(76.6 \%)$ & 185 & $173(86.0 \%)$ \\
\hline Heart & 340 & $254(80.8 \%)$ & 196 & 175 (89.2 \%) \\
\hline Intestinal lymph & 433 & $256(76.6 \%)$ & 220 & $160(86.4 \%)$ \\
\hline Kidney & 943 & $241(70.8 \%)$ & 551 & $156(79.5 \%)$ \\
\hline Leg muscle & 252 & $550(58.3 \%)$ & 129 & $386(70.0 \%)$ \\
\hline Liver & 872 & $186(73.8 \%)$ & 524 & 97 (75.1 \%) \\
\hline Lung & 1124 & $506(58.0 \%)$ & 711 & $363(69.2 \%)$ \\
\hline Mammary gland & 187 & $588(52.3 \%)$ & 100 & $443(62.3 \%)$ \\
\hline Ovary & 254 & $293(67.6 \%)$ & 131 & $162(73.6 \%)$ \\
\hline Skin black & 536 & $147(78.6 \%)$ & 307 & $82(82 \%)$ \\
\hline Skin white & 440 & 199 (78.3 \%) & 268 & $108(82.4 \%)$ \\
\hline Spleen & 260 & $196(75.3 \%)$ & 126 & $102(80.9 \%)$ \\
\hline Thymus & 132 & $108(81.8 \%)$ & 79 & 71 (89.8 \%) \\
\hline Thyroid & 470 & $383(81.4 \%)$ & 264 & $227(85.9 \%)$ \\
\hline Tongue & 216 & $174(80.5 \%)$ & 111 & 96 (86.4 \%) \\
\hline White blood cells & 325 & $327(74.3 \%)$ & 201 & $215(91.4 \%)$ \\
\hline
\end{tabular}

to what they were for gene expression, i.e., heart does not cluster with the other muscle samples for ASE, while it did for gene expression, indicating tissue specific differences in ASE. Interestingly lung, liver and kidney show very little correlation of ASE with any other tissues or with each other, these tissues showed the highest levels of ASE. Of the 4033 genes expressed in more than one tissue showing ASE, and where the major allele's parental origin could be determined (see Methods), 2324 (58 \%) genes showed divergent allele specific patterns, that is the major allele expressed was not the same across all tissues. This was previously demonstrated in Additional file 1: Figure S3A. An example of a single SNP is given in Fig. 6a for a SNP in gene $S P T Y 2 D 1$, where the paternal allele is expressed in kidney and thymus (0.82 and 0.92 paternal allele frequency respectively) and maternal allele in the brain caudal lobe and brain cerebellum (0.12 and 0.08 paternal allele frequency respectively). Mutations in SPTY2D1 (Suppressor of Ty, domain containing 1) have been associated with lipid levels in humans in multiple studies $[17,18]$, and interestingly Guo et al. [18] suggested this association might be sex specific. Additional file 1: Figure S3B, C, D and E shows examples of ASE patterns across tissues for multiple SNP within the genes GBP5, PRUNE2, SGOL2 and SAMD9. They demonstrate that allele specific expression patterns within genes and across tissues can be extremely divergent and complex, when looking at multiple SNP in many tissues.
Tissue specific ASE (TS ASE) has been reported previously. One of the most extensive tests, by Keane et al. [19], performed RNAseq in a single F1 mouse and tested 6975 genes across 6 tissues and found that $95 \%$ of genes showing ASE did so in a tissue specific manner. Another by Pinter et al. [20] performed RNAseq in a reciprocal cross between two mouse strains, testing 7465 genes across two tissues and found $82 \%$ of ASE genes showed TS ASE. Keane et al. [19] and Pinter et al. [20] also found that 12 and $21 \%$ of ASE genes showed divergent allele specific patterns.

Interestingly we found that there are ten genes that show ASE exclusively in mammary gland, ZMYND11, CLSPN*, SLC3OA2*, TECTB*, SERTAD2, ZNF638, $A L D H 3 B 2 \%, C I D E A^{*}, T F F 1^{*}$ and one uncharacterized protein*. Seven of these genes are differentially expressed when compared with all other tissues (denoted with * above) and all seven are up regulated. These genes have functions associated with mammary development, involution, or milk nutrition; ZMYND11, CLSPN, TFF1, affect proliferation or apoptosis of mammary cells [21-23]; SLC30A2 transports Zinc from the mammary gland into milk [24]; CIDEA is a transcriptional coactivator regulating mammary gland secretion of milk lipids [25]; and TFF1 is a gastrointestinal protective peptide secreted into milk [26]. Genes that show ASE exclusively in one tissue are often the genes that are differentially expressed, when comparing that tissue with all other 

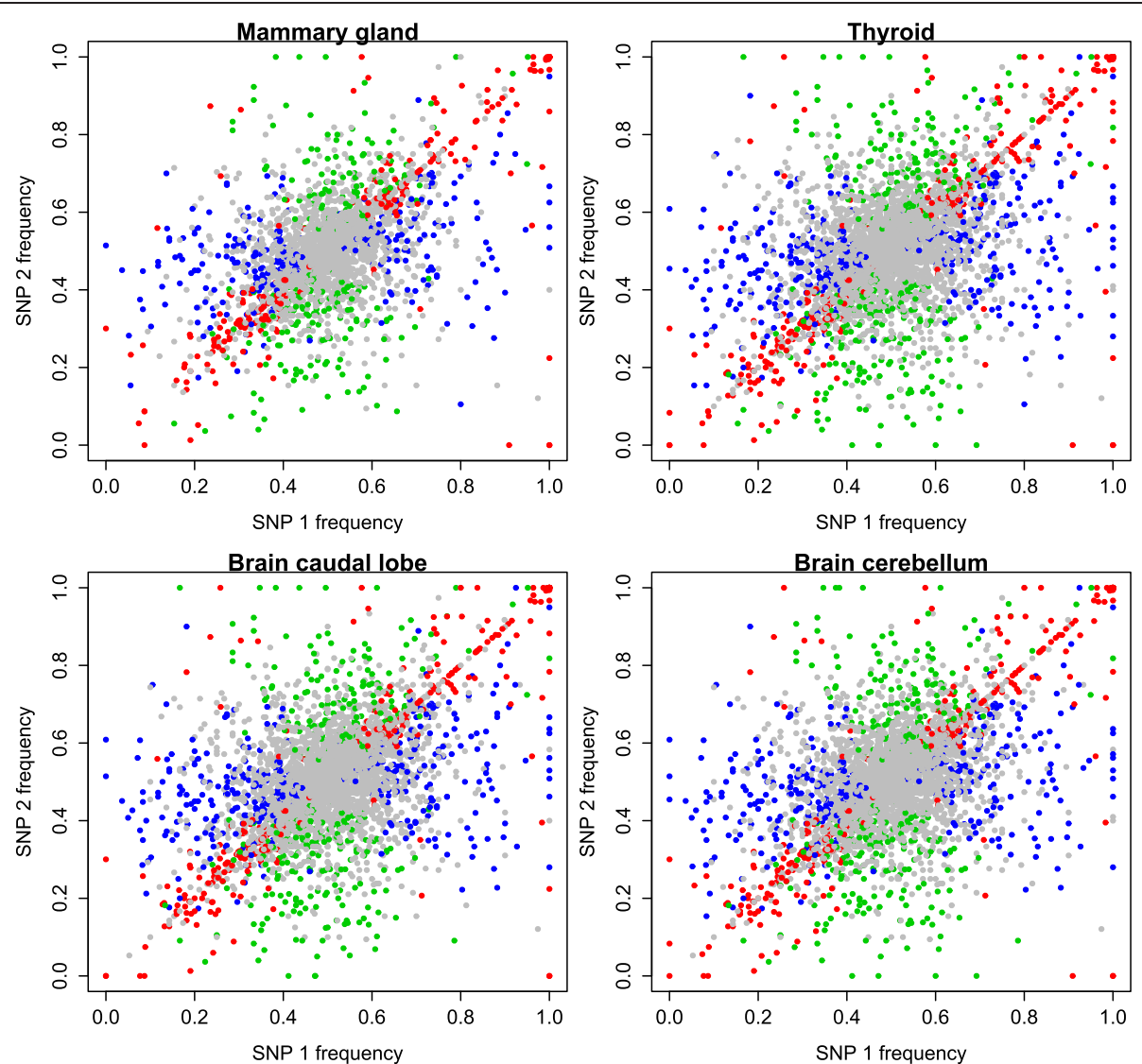

Fig. 4 Using only SNP that could be definitively phased and that occurred in genes with 2 or more SNP, these plots for mammary gland, thyroid, brain caudal lobe and brain cerebellum show the paternal allele frequency for neighbouring SNP within a gene. Red points are those where both SNP were significantly ASE, blue points are those where only SNP1 was significantly ASE, green points are those where only SNP2 was significantly ASE, grey points are where neither SNP was significantly ASE

tissues (Additional file 1: Table S9). This is a theme which is consistent across all tissues (Additional file 1: Table S9). This could simply be because we have greater power to detect ASE in highly expressed genes. However, it may also be that genes that are highly expressed in a particular tissue are likely to be regulated by multiple sites in the surrounding DNA and these sites are likely to contain at least one SNP which is heterozygous. All genes that show exclusive TS ASE are listed in Additional file 2: Table S10. There were some highly expressed genes that did not show significant ASE, such as alpha s2 casein (CSN1S2) and beta casein (CSN2) which are two of the major milk proteins highly expressed in mammary gland (Additional file 1: Table S11), indicating that our test does not always find ASE in highly expressed genes.

Monoallelic expression (MAE) in at least one tissue was observed in 4298 of 7985 genes tested (54\%) and for 1349 of 4856 genes tested with more than one heterozygous SNP with ASE (28\%, Table 3). We have categorised ASE as MAE where the major allele is at a frequency $>90 \%$. This category of genes is the most affected by errors in the cow's genome sequence (i.e. Fig. 3) and so we consider the
$28 \%$ of genes with MAE for more than one SNP to be most likely to be MAE (as opposed to genes with MAE for just a single SNP), but that the true estimate is somewhere between 28-54 \%. Within tissue estimates of genes that are MAE in this study range from $4.1-5.8 \%$ in thymus and thyroid and $24-38 \%$ in lung with an average of 7-12\% across all tissues. The correlation of MAE between tissues (Fig. 5b) shows some correlation between their MAE patterns but largely these expression patterns are more different between tissues than ASE (compare Fig. $5 \mathrm{~b}$ and a). Our conservative estimate of $28 \%$ of genes showing MAE across tissues is higher than reported by Keane et al. [19] in 6 mouse tissues who found that $12 \%$ of genes showed significant extreme (defined as $>75 \%$ major allele) allelic bias and Pinter et al. [20] who found that $5 \%$ of genes tested showed monoallelic expression (defined as $100 \%$ major allele) in 2 mouse tissues. However in this study we have tested many more tissues than either of those studies. Our estimates of 7-12\% within single tissues agree with published reports - Gimelbrant et al. [27] reported that 5-10 \% of 4000 tested autosomal genes displayed random monoallelic expression in human 


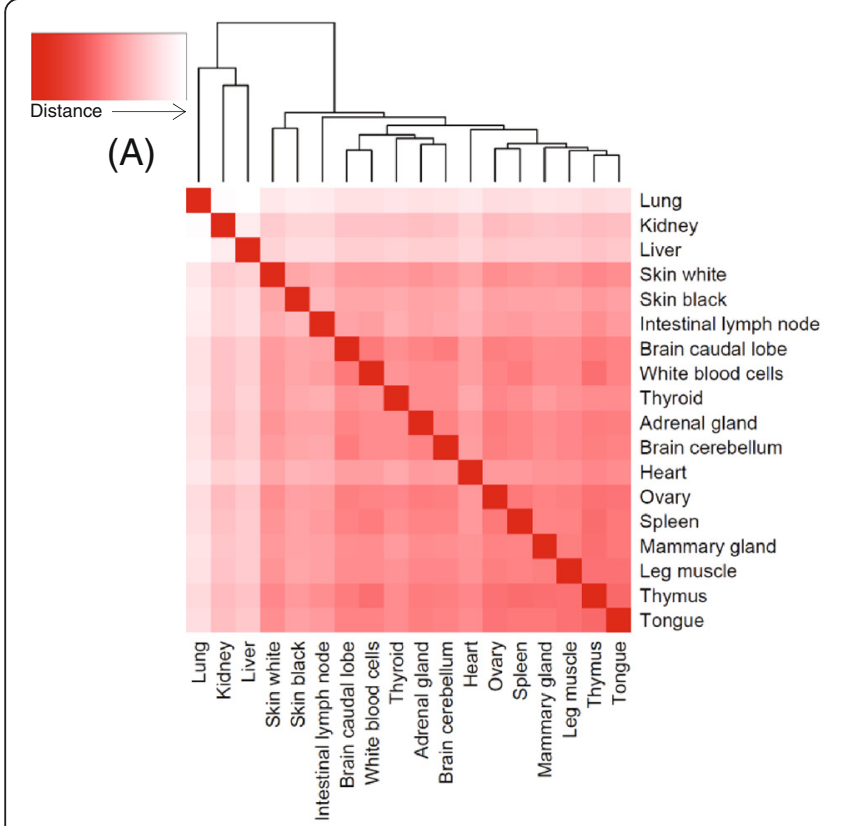

(B)

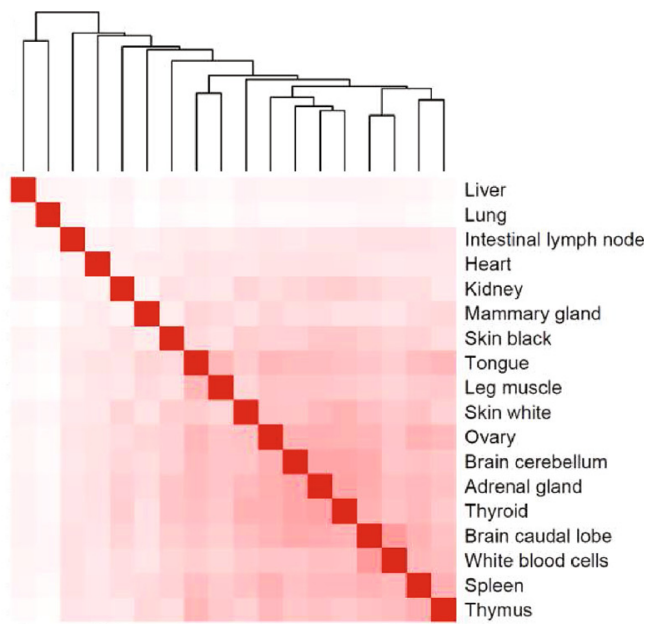

(C)

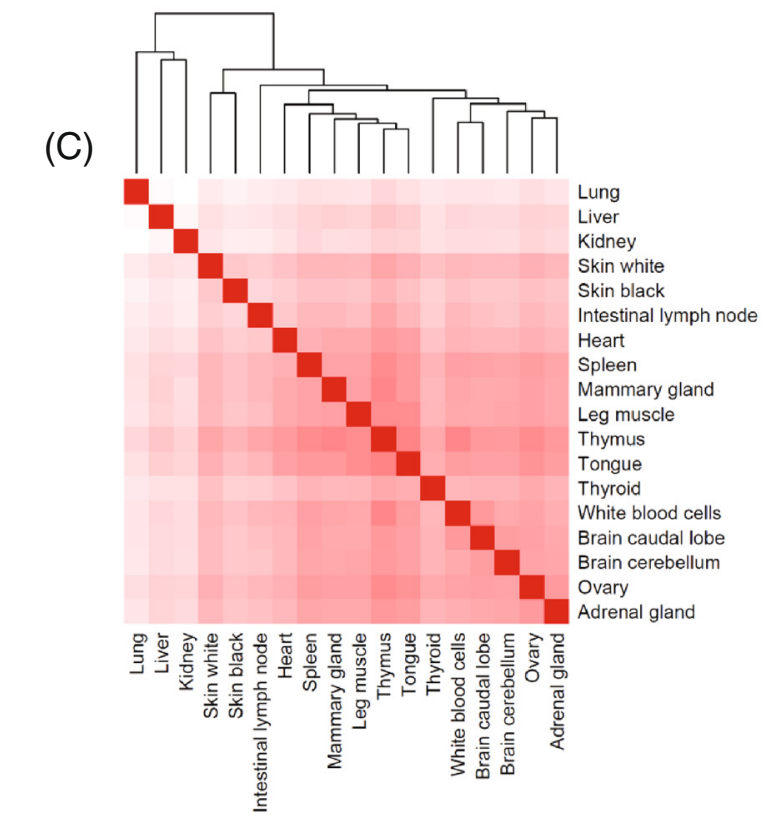

(D)
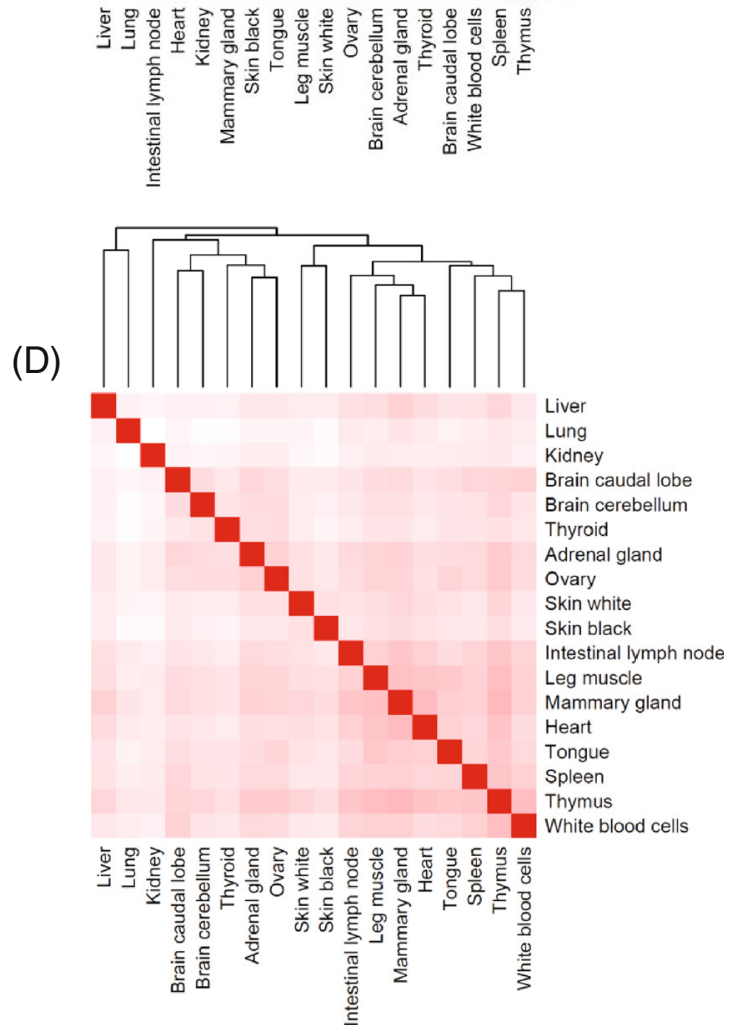

Fig. 5 Heat maps of tissue-to-tissue distances generated by applying the 'dist' function in $\mathrm{R}$ to the transpose of a matrix, as defined for each heat map. For each heat map, the variability between tissues is measured by the height of the dendrogram branches. The colour key indicates the distance between tissues with red being the least distant (or most correlated) and white being the most distant (or least correlated). a Uses a SNP by tissue matrix containing the paternal allele frequency of all ASE SNP, therefore displaying the correlation of ASE between tissues. $\mathbf{b}$ Uses a SNP by tissue matrix in which SNP were assigned " 1 " if mono-allelic and " 0 " if not, therefore displaying the correlation of MAE between tissues. c Uses a gene by tissue matrix in which genes classified as maternal were assigned "-0.5", genes classified as paternal were assigned a value of " 0.5 " and genes that could not be classified or were not expressed were assigned " 0 ", therefore displaying the correlation of paternal ASE between tissues. d Uses a gene by tissue matrix in which all genes in "runs" of 5 or more (as defined in methods) were designated a value of "1" and genes not in a run were designated " 0 ", therefore displaying the between tissue correlation of genes falling into runs of greater than 5 or more expressing the same allele

clonal cell lines, and Zwemer et al. [28] report that $10 \%$ of 1358 autosomal genes tested showed random monoalleleic expression in mouse cell lines. Our estimates of 7-12\% within single tissues agree with these published reports.
Validation of allele specific and mono allelic expression In an attempt to confirm some of the above findings we tested ASE in a validation dataset. This data consisted of RNA sequence reads from 20 first lactation Holstein dairy cows for two tissues, liver and white blood cells 


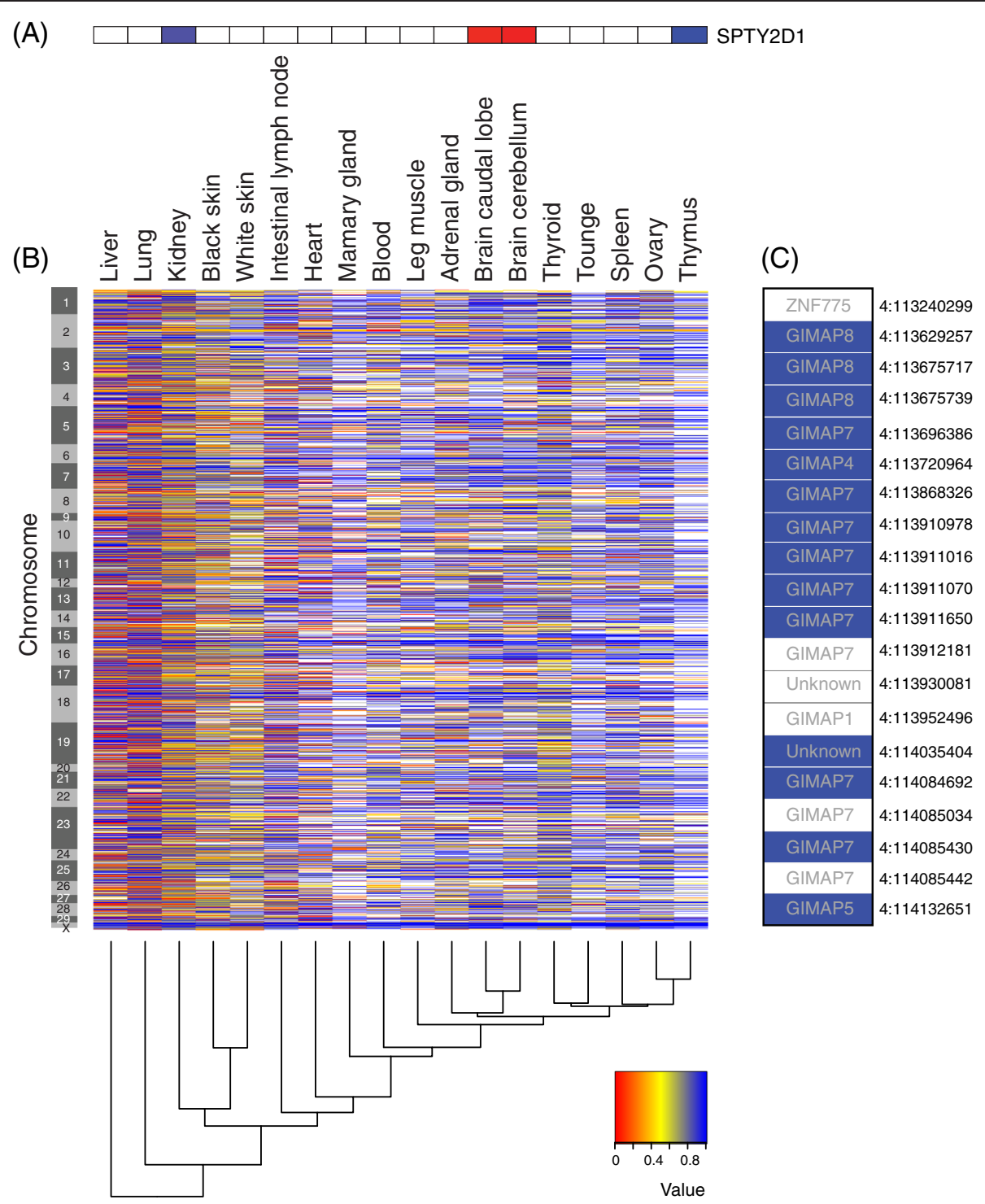

Fig. 6 Using only SNP that could be definitively phased, (a) is a heat map of the paternal allele frequency of the same SNP on chromosome 29 (in SPTY2D1) across all 18 tissues. The frequency of paternal expression is scaled from 0 (100\% maternal, red) to 1 (100 \% paternal, blue). b A heat map of the frequency of the paternal allele for all phased SNP, for each tissue. Tissues are clustered on the overall paternal expression as indicated by the dendrogram. SNP are ordered by position and the left hand scales indicate the different (ordered) chromosomes. c Highlights 14 SNP within 5 known genes (GIMAP8, GIMAP7 copy 1, GIMAP4, GIMAP7 copy 2 and GIMAP5, listed within each cell) on chromosome four in the brain caudal lobe, chromosome and position are listed on the right hand side of the expression pattern

(WBC). The data was mapped and tested for ASE in a similar manor to the original dataset above (see Methods for details). However these animals did not have whole genome sequence data and so greater than 28 million SNP were imputed for these animals and phased genotypes used to create parental genomes and then tested for ASE in the RNA sequence reads. On average 19 million reads were generated per library with $82.8 \%$ passing quality control, and $89 \%$ of those mapped uniquely to both the maternal and paternal genomes (Additional file 1: Table S12).
On average, across the 20 samples, $13-29 \%$ of the 3531 genes tested in white blood cells and $12-26 \%$ of the 2939 genes tested in liver showed significant ASE (Additional file 1: Table S13). However across all samples $35-72 \%$ of 6521 genes tested in white blood cells and 31-65\% of 8187 genes tested in liver showed significant ASE (Additional file 1: Table S13). That means that 72 and $65 \%$ of all genes tested across all samples showed significant ASE in white blood cells and liver respectively, in at least one animal. The within sample estimates in white blood cells agreed very well 
Table 3 Monoallelic expression results, the number and proportion of SNP tested that were showing monoallelic expression (MAE SNP), that is the major allele is at a frequency $>90 \%$, in each tissue and in total. Also the number and proportion of genes tested that contained MAE SNP (Genes w/ MAE SNP). Then the number and proportion of genes with greater than one SNP showing MAE (Genes w/ 1 MAE SNP) in each tissue and in total

\begin{tabular}{llll}
\hline Tissue & MAE SNP (\% tested) & Genes w/ MAE SNP (\% tested) & Genes W/ >1 MAE SNP (\% tested) \\
\hline Adrenal gland & $690(5 \%)$ & $370(6.8 \%)$ & $144(4.6 \%)$ \\
Brain caudal lobe & $776(5 \%)$ & $398(6.7 \%)$ & $164(4.7 \%)$ \\
Brain cerebellum & $665(4 \%)$ & $340(6.0 \%)$ & $139(4.3 \%)$ \\
Heart & $994(10 \%)$ & $702(17.6 \%)$ & $197(9.3 \%)$ \\
Intestinal lymph & $1236(11 \%)$ & $881(18.8 \%)$ & $237(9.3 \%)$ \\
Kidney & $1362(8 \%)$ & $859(14.5 \%)$ & $266(7.7 \%)$ \\
Leg muscle & $564(5 \%)$ & $361(8.1 \%)$ & $106(4.3 \%)$ \\
Liver & $2333(19 \%)$ & $1540(31.5 \%)$ & $484(17.8 \%)$ \\
Lung & $3215(23 \%)$ & $2080(38.4 \%)$ & $726(23.9 \%)$ \\
Mammary gland & $544(7 \%)$ & $373(10.5 \%)$ & $108(5.9 \%)$ \\
Ovary & $603(4 \%)$ & $349(6.2 \%)$ & $128(4.0 \%)$ \\
Skin black & $1032(6 \%)$ & $680(11.6 \%)$ & $186(5.5 \%)$ \\
Skin white & $870(5 \%)$ & $501(8.3 \%)$ & $173(4.9 \%)$ \\
Spleen & $627(4 \%)$ & $372(7.0 \%)$ & $126(4.1 \%)$ \\
Thymus & $429(4 \%)$ & $230(5.8 \%)$ & $89(4.1 \%)$ \\
Thyroid & $750(4 \%)$ & $361(5.8 \%)$ & $153(4.1 \%)$ \\
Tongue & $531(4 \%)$ & $321(6.6 \%)$ & $108(4.0 \%)$ \\
White blood cells & $803(6 \%)$ & $401(8.6 \%)$ & $171(6.4 \%)$ \\
Total & $8546(34 \%)$ & $4298(53.8 \%)$ & $1349(27.8 \%)$ \\
\hline
\end{tabular}

with the 21-33\% of genes that showed ASE in the discovery dataset (Table 1). Within sample estimates in liver were dramatically lower than that of the 59-73\% of genes that showed ASE in the discovery dataset (Table 1). However on average samples in the validation dataset had only 15 million paired reads mapped and tested only 2939 genes, compared with the discovery dataset that had 102 million paired reads mapped and tested 4887 genes for liver, therefore this reduction in the proportion of genes showing ASE is likely a reflection of the reduced sequence coverage and therefore power to detect ASE in individuals within the validation dataset.

On average, across all 20 samples, $0.6-4.3 \%$ of 3531 genes and $0.7-4 \%$ of 2939 genes tested for white blood cells and liver respectively showed significant MAE (Additional file 1: Table S14). However across all samples $2.5-22 \%$ of 8970 genes and $2.6-20 \%$ of 8187 genes tested in white blood cells and liver respectively showed significant MAE respectively (Additional file 1: Table S14). These within sample estimates were much lower than the $6.4-8.6$ and $17.8-31.5 \%$ of genes tested in white blood cells and liver respectively of the discovery dataset. However again we attribute this to the sequence coverage and therefore the lower power to detect ASE (including MAE) in the individual validation samples.
Interestingly when we combined samples within the validation dataset the proportion of genes that showed ASE increased to 35-72 and 31-65\% in white blood cells and liver respectively (Additional file 1: Table S13). Also the proportion of genes that show MAE increased to 2.5-22 and 2.6-20\% in white blood cells and liver respectively (Additional file 1: Table S14). This reflects the variation in the genes that show ASE across the 20 animals (Additional file 1: Figure S4) and the moderate correlation between individuals (within tissue) of genes showing ASE (Additional file 1: Figure S5). From Additional file 1: Figure S4 we can see that there are only 1847 of the 6298 genes that showed ASE (Additional file 1: Table S14) in five or more of the 20 animals in WBC, and 1248 of the 5169 genes in liver. These drop to 519 and 370 for WBC and liver respectively in ten or more of the samples. For the validation dataset only, the correlation of genes showing ASE is higher within tissues (Additional file 1: Figure S5), however the correlations are still only moderate, reflecting the variability in genes that display ASE across multiple individuals. Additional file 1: Figure S5 also shows that when the same tissue samples from the discovery dataset are included, the two tissues from the discovery dataset are more correlated with each other than they are with the other samples from the same tissues. We hypothesis that this difference is a reflection 
of the number of genes able to be used in the correlation calculation, therefore the power of the test is greater for that comparison. This relationship was also reported by the GTEx consortium [7]. They compared between-sample, and between-tissue, sharing of ASE with overall similarity of gene expression. They found that gene expression levels were similar between individuals when comparing within tissue, and different between tissues when comparing within individual gene expression, as you would expect. However allelic ratios had a higher correlation among tissues from the same individuals than among different individuals for the same tissue, suggesting that ASE is primarily determined by the individual's genome. Therefore by testing only one individual we have likely underestimated the total number of genes displaying ASE in the cattle population, and further testing in more individuals will uncover more genes that show ASE.

\section{Preferential expression of the paternal allele}

Using the SNP with known parental origin, we were able to classify genes as expressing either the paternal or maternal allele. Our results found that on average $54.17 \%$ of all genes within a tissue, with significant ASE SNP and where parental origin could be determined, favoured expression of the paternal allele (Table 4). The proportion of genes expressing the reference allele was on average $50.76 \%$, a difference of $3.41 \%$. Using a t-test we found there was significantly $(p=0.04)$ more paternal expression than reference allele expression (with pairs being tissues). The distribution of all genes where parental origin could be determined (not just those containing SNP significantly ASE) is depicted in Additional file 1: Figure S6. It shows that on average across all tissues $13.3 \%$ of all genes tested express both alleles equally, $39.7 \%$ express the maternal allele preferentially and $46.9 \%$ express the paternal allele preferentially. This can also be seen in the heat map of allele expression (Fig. 6b), where the majority of tissues are dominated by paternal allele expression (blue). The tissues are also clustered on the $\mathrm{x}$ axis by the alleles that are expressed, so that the tissues on the left have more maternal expression (yellow) but, as we move across the tissues to the right, the amount of maternal expression decreased. However when SNP with paternal allele frequency of zero or one were removed, $50.74 \%$ of all genes favoured expression of the paternal allele. This indicates that it is the genes that show MAE that are favouring the paternal allele. The correlation of this paternal expression between tissues is shown in Fig. 5c, which demonstrated correlation between tissue's paternal ASE patterns, but largely these expression patterns are more different between tissues than ASE (Fig. 5a). Crowley et al. [6] reported that $54-60 \%$ of all genes, with significant ASE, favoured expression of the paternal allele in mouse brain. This is consistent with our estimates of $53-57 \%$ in brain caudal lobe and in brain cerebellum respectively (Table 4). Because we were only able to determine phase in $50 \%$ of the SNP tested we have not been able to test as many genes as was tested by Crowley et al. [6]. We have however tested many more tissues and found the same allele expression pattern favouring the paternal allele in many tissues (Table 4).

There is now accumulating evidence that expression within cells is from one chromosome (paternal or maternal). Borel et al. [29] tested ASE in 203 single cells. They found that for most actively transcribed genes one allele was predominantly expressed in each of the single cells, and that equal numbers of single cells expressed one or the other allele, with very few (less than $5 \%$ of cells) expressing both alleles. Pinter et al. [20] performed RNA-DNA FISH and RNAseq on mouse tail fibroblast cells. RNA-DNA FISH showed that the majority of single cells expressed only a single allele. Also, for genes that showed ASE in the RNAseq data, there was an imbalance in the number of cells expressing the major allele. So if this is the case in all mammals then what we see in this study is that within certain tissues there are more cells within a tissue complex expressing the paternal allele at any one time for genes that show MAE.

\section{Coordinated expression of neighbouring genes}

To investigate whether there was evidence for coexpression of genes in close proximity to each other, we performed a Wald-Wolfowitz runs test on an ordered list of genes classified by paternal or maternal expression in each tissue (Table 4). Our results show that nine of the 18 tissues displayed significant $(p<0.05)$ clustering of expression, greater than expected by chance. This means that neighbouring genes were more likely to express the allele from the same chromosome (maternal or paternal). Figure $5 \mathrm{~d}$ shows the correlation between tissues of clusters with greater than five genes expressing the same allele and Additional file 1: Figure S7 shows the frequency of runs of 2-40 genes showing the same parental inheritance. For brain caudal lobe, one chromosome region showing expression from the same chromosome included 14 SNP across the five known genes, GIMAP8, GIMAP7 copy1, GIMAP4, GIMAP7 copy 2 and GIMAP5 that all detect strong ASE of the paternal allele (Fig. 6c). These five known genes are from one gene family known as GIMAP family (GTPase, IMAP Family) and they have no known common control region. The function for these genes in brain is not known but previous studies have found these genes predominantly expressed in mature lymphocytes, and suggest they have a role in lymphocyte survival [30]. 
Table 4 The total number of genes showing significant ASE, where phase could be determined, and the number and proportion of those where the major allele is the paternal allele and the reference allele

\begin{tabular}{|c|c|c|c|c|}
\hline Tissue & ASE genes classified & Paternal genes (\%) & Reference genes (\%) & WW runs test ( $p$-value) \\
\hline Adrenal gland & 1189 & 594 (49.95\%) & 647 (54.41\%) & $0.0006^{*}$ \\
\hline Brain caudal lobe & 1038 & $558(53.75 \%)$ & $530(51.05 \%)$ & 0.54 \\
\hline Brain cerebellum & 1026 & $582(56.72 \%)$ & 497 (48.44 \%) & $0.0063^{*}$ \\
\hline Heart & 1360 & 710 (52.20 \%) & $702(51.61 \%)$ & 0.27 \\
\hline Intestinal lymph & 1645 & 873 (53.06 \%) & $862(52.40 \%)$ & 0.40 \\
\hline Kidney & 3490 & 1807 (51.77 \%) & $1718(49.22 \%)$ & $0.001^{*}$ \\
\hline Leg muscle & 910 & 473 (51.97 \%) & 457 (50.21\%) & 0.5 \\
\hline Liver & 3236 & $1672(51.66 \%)$ & $1633(50.46 \%)$ & 0.18 \\
\hline Lung & 4321 & $2286(52.90 \%)$ & $2179(50.42 \%)$ & $0.04^{*}$ \\
\hline Mammary gland & 721 & 370 (51.31\%) & $346(47.98 \%)$ & 0.66 \\
\hline Ovary & 854 & 488 (57.14 \%) & $422(49.41 \%)$ & 0.09 \\
\hline Skin black & 2058 & $1114(54.13 \%)$ & 1048 (50.92 \%) & $0.009^{*}$ \\
\hline Skin white & 1587 & 889 (56.01\%) & 809 (50.97 \%) & $0.018^{*}$ \\
\hline Spleen & 897 & $513(57.19 \%)$ & $436(48.60 \%)$ & 0.27 \\
\hline Thymus & 398 & $240(60.30 \%)$ & $221(55.52 \%)$ & 0.11 \\
\hline Thyroid & 1483 & 808 (54.48 \%) & 753 (50.77 \%) & $0.00018^{*}$ \\
\hline Tongue & 749 & 437 (58.34 \%) & $365(48.73 \%)$ & 0.11 \\
\hline White blood cells & 1101 & $575(52.22 \%)$ & $579(52.58 \%)$ & $0.03^{*}$ \\
\hline Average & & $54.17 \%$ & $50.76 \%$ & \\
\hline
\end{tabular}

Note phase could not be determined for all SNP tested. Also $p$-value from the Wald-Wolfowitz (WW) runs test in each tissue, where ${ }^{*}$ denotes those that are significant

Crowley et al. [6] also found that genes with higher expression from one parental allele tended to cluster in mouse. They found that, among the 19 autosomes, 15 had a higher proportion of genes whose neighbour had the same parental skew in expression which was more than expected by chance.

\section{Allele specific expression in known imprinted genes}

One source of ASE is imprinting, so we investigated whether genes previously found to be imprinted in mammals (humans, mice or cattle), extracted from the gene imprint database (http://www.geneimprint.com/ site/home), showed extreme ASE in our data set. Thirty one of these genes, four of which had been previously reported to be imprinted in cattle (NAPIL5, SLC22A3, RTL1, Igf2r), could be tested for imprinting in our data because there was both expression of the gene (at least 10 reads) and a heterozygous SNP in the transcripts from that gene. Genes were classified as either not imprinted, imprinted, partially imprinted, tissue specifically imprinted or tissue specific partially imprinted (Table 5). There was evidence for ASE in 17 genes out of the 31 tested, including all four previously reported as imprinted in cattle (Table 5). Twelve of the 17 genes showed ASE in specific tissues, varying from one of the tissues showing expression (e.g., COPG2IT1), to all but one of the tissues showing expression (e.g., IGF2) (Table 6 and Additional file 1: Table S15). Six of the 17 genes had the same allele expressed as was previously reported, including all 4 genes previously reported as imprinted in cattle (Table 6). Seven had the opposite allele expressed but none of these genes had previously been reported as imprinted in cattle (Table 6). For example Adenosine monophosphate deaminase 3 (AMPD3) was reported as expressing the maternal allele in mice, however our data suggests tissue dependant ASE. Here we found the paternal allele expressed in thyroid and blood, and the maternal allele expressed in lung. Bos Taurus growth factor receptor-bound protein 10 (GRB10) imprinting was reported as isoform dependant in human and mouse, here in cattle we found partial imprinting of just the paternal allele in all 11 tissues.

Because we could distinguish maternal and paternal alleles only in one animal, we were unable to distinguish imprinting from cis eQTL in this study. However, it seems likely that the ASE we observed in the four genes previously reported to be imprinted in cattle (NAPIL5, $S L C 22 A 3, R T L 1, I g f 2 r)$ is due to imprinting. The results for other genes could be due to cis eQTL or they may indicate that imprinting is a more variable phenomenon than classically described and varies in extent and tissue specificity. 
Table 5 Summary of classification of genes previously found to be imprinted in human and mice or cattle

\begin{tabular}{|c|c|c|c|c|c|}
\hline Class & ASE & MAF $>90 \%$ & All tissues & Human \& mouse & Cattle \\
\hline Not Imprinted & No & No & Yes & 14 & 0 \\
\hline Imprinted & Yes & Yes & Yes & 2 & 2 \\
\hline Partially Imprinted & Yes & No & Yes & 1 & 0 \\
\hline Tissue Specifically Imprinted & Yes & Yes & No & 3 & 2 \\
\hline Tissue Specific Partially Imprinted & Yes & No & No & 7 & 0 \\
\hline Total tested & & & & 27 & 4 \\
\hline Total imprinted & & & & 13 & 4 \\
\hline
\end{tabular}

The number of genes classified as either not imprinted, imprinted, partially imprinted, tissue specifically imprinted or tissue specific partially imprinted. ASE defines whether the class of genes showed significant ASE, MAF $>90 \%$ defines if the major allele frequency was greater than $90 \%$ and All tissues defines if the ASE result was observed across all tissue the gene was expressed in

\section{Implications for cattle breeding}

Our attention must now turn to the identification of these cis-regulatory variants, and the international FAANG (Functional Annotation of Animal Genomes) consortium [31] aims to do this for livestock species. The identification of causative regulatory variants could then be used in livestock genomic selection programs leading to more accurate genomic breeding values and increases in the rate of genetic gain for economically important traits in cattle and other livestock species. A promising example where this has been tried has been in chicken's response to Mareks disease infection, [32, 33]. The authors discovered SNP that showed ASE in response to infection and found they accounted for $\sim 83 \%$ of the genetic variance for Mareks disease resistance [32]. They then used those SNP to genotype a resource population of 1000 chickens to generate SNP effects for Mareks disease resistance, and used those in

Table 6 Genes found to be imprinted in this dataset, along with gene identifiers, which species and which allele was previously reported, the number of SNP used to test for imprinting, the number of tissues the SNP were expressed in, the number of tissues where the SNP showed imprinting or partial imprinting, the imprinting status and expressed allele in this dataset. The expressed allele was determined from the SNP tested or where they were not informative from other SNP within the gene but not within exons or from SNP flanking the gene

\begin{tabular}{|c|c|c|c|c|c|c|c|c|c|}
\hline $\begin{array}{l}\text { Bovine } \\
\text { ensembl ID }\end{array}$ & $\begin{array}{l}\text { Gene } \\
\text { name }\end{array}$ & $\begin{array}{l}\text { Expressed allele } \\
\text { reported }\end{array}$ & $\begin{array}{l}\text { Species } \\
\text { reported }\end{array}$ & \#SNP & $\begin{array}{l}\text { \#Tissues } \\
\text { expressed }\end{array}$ & $\begin{array}{l}\text { \#Tissues } \\
\text { imprinted }\end{array}$ & $\begin{array}{l}\text { \#Tissues partially } \\
\text { imprinted }\end{array}$ & $\begin{array}{l}\text { Imprinting } \\
\text { status }\end{array}$ & $\begin{array}{l}\text { Expressed } \\
\text { allele }\end{array}$ \\
\hline ENSBTAG00000010128 & NAP1L5 & Paternal & human, mouse, cattle & 3 & 13 & 13 & 0 & 1 & \\
\hline ENSBTAG00000018645 & $D L X 5$ & Maternal & human & 1 & 1 & 1 & 0 & 1 & Paternal \\
\hline ENSBTAG00000039080 & $S L C 22 A 3$ & Maternal & human, mouse & 1 & 5 & 5 & 0 & I & Maternal \\
\hline ENSBTAG00000046585 & RTL 1 & Paternal & human, mouse, cattle & 3 & 1 & 1 & 0 & 1 & Paternal \\
\hline ENSBTAG00000047473 & NLRP2 & Maternal & human & 8 & 1 & 0 & 1 & $\mathrm{Pl}$ & Paternal \\
\hline ENSBTAG00000002402 & $\lg 22 r$ & Maternal & mouse, cattle & 2 & 14 & 4 & 1 & TSI & Maternal \\
\hline ENSBTAG00000008361 & Pon2 & Maternal & mouse & 3 & 14 & 0 & 1 & TSPI & Paternal $^{\mathrm{a}}$ \\
\hline ENSBTAG00000013066 & $\lg f 2$ & Paternal & mouse, cattle & 1 & 13 & 12 & 0 & TSI & Paternal \\
\hline ENSBTAG00000017245 & COPG2IT1 & Paternal/Maternal & human, mouse & 1 & 14 & 1 & 0 & TSI & Paternal $^{a}$ \\
\hline ENSBTAG00000024426 & PPP1R9A & Maternal & human, mouse & 2 & 9 & 2 & 0 & TSI & Paternal \\
\hline ENSBTAG00000027081 & ATP10A & Maternal & human, mouse & 13 & 9 & 0 & 4 & TSPI & Paternal $^{a}$ \\
\hline ENSBTAG00000034645 & Pon3 & Maternal & mouse & 1 & 13 & 5 & 0 & TSI & Paternal $^{b}$ \\
\hline ENSBTAG00000002813 & Gab1 & Paternal & mouse & 2 & 18 & 0 & 1 & TSPI & \\
\hline ENSBTAG00000003035 & Impact & Paternal & mouse & 18 & 18 & 0 & 17 & TSPI & Paternal \\
\hline ENSBTAG00000006640 & $R B 1$ & Maternal & human & 4 & 18 & 0 & 4 & TSPI & Paternal ${ }^{\text {ab }}$ \\
\hline ENSBTAG00000015821 & Ampd3 & Maternal & mouse & 6 & 12 & 0 & 3 & TSPI & $\begin{array}{l}\text { Tissue } \\
\text { Specific }^{c}\end{array}$ \\
\hline ENSBTAG00000017086 & GRB10 & Isoform dependant & human/mouse & 8 & 16 & 0 & 11 & TSPI & Paternal \\
\hline
\end{tabular}

${ }^{\mathrm{a}}$ Based on SNP within gene boundaries but not within exon

based on SNP upstream of gene

${ }^{c}$ Based on SNP up and downstream of gene. Lung maternal, thyroid and lymph node paternal

Imprinting status is defined as: I Imprinted, PI Partially imprinted, TSI Tissue specifically imprinted, TSPI Tissue specific partially imprinted 
the genomic prediction of 60 roosters. These roosters were progeny tested for Mareks disease resistance. The genomic predictions based on the ASE SNP had $61 \%$ higher accuracy than traditional breeding values [33]. So although the SNP used were unlikely to be causative they would have been in high LD with the causative regulatory variants, resulting in them being highly predictive of Mareks disease resistance. This approach could be used in cattle for important diseases such as tuberculosis and trypanosomiasis. The identification of causative regulatory variants is expected to further increase the accuracy of genomic predictions for production and health traits.

\section{Conclusion}

Investigating the extent of ASE across 18 bovine tissues in one cow and two tissues in 20 cows demonstrates 1) ASE is pervasive in cattle, 2) the ASE is often MAE but ranges from MAE to slight overexpression of the major allele, 3) the ASE is most often tissue specific and that more than half the time displays divergent allele specific expression patterns across tissues 4) the expression slightly favours the paternal allele and 5) genes expressing the same parental allele are clustered together more than expected by chance, and there are several runs of large numbers of genes expressing the same parental allele.

\section{Methods}

\section{Tissue sampling}

Eighteen tissues: black skin, white skin, adrenal gland, brain caudal lobe, brain cerebellum, heart, kidney, liver, lung, intestinal lymph node, mammary, leg muscle (semimembranosus), ovary, spleen, thymus, thyroid and tongue, from one lactating dairy cow were collected directly after euthanasia, whole blood was collected by venipuncture of the coccygeal vein just prior to euthanasia. The cow was 25 months old and 65 days into her first lactation and was euthanized because she injured her rear leg not for the purposes of this study, for this reason the local Animal Ethics Commitee (DEPI Agricultural Research and Extension Animal Ethics Commitee) advised ethics approval was not required. Tissues were dissected by a veterinarian and then into $\sim 100 \mathrm{mg}$ tissue samples and snap frozen in liquid nitrogen. Tissues were stored at $-80 \mathrm{oC}$. Blood was processed according to the blood fractionation and white blood cell stabilisation procedure in the RiboPure ${ }^{\mathrm{Tx}}$ blood kit (Ambion by Life Technologies) protocol. White blood cells in RNA later were then stored at $-20 \mathrm{oC}$.

\section{RNA extraction, library preparation and sequencing}

One hundred $\mathrm{mg}$ of each tissue was ground in triplicate using a TissueLyserII (Qiagen) and liquid nitrogen. RNA was extracted from $\sim 30 \mathrm{mg}$ of ground tissue using Trizol (Invitrogen) according to standard protocol. RNA was then passed through an RNeasy column (Qiagen) and eluted in 30ul RNase free water. RNA was extracted from stabilised white blood cells using RiboPure ${ }^{\text {tn }}$ blood kit according to protocol. RNA quality was assessed on BioAnalyser 2100 (Agilent Technologies). RNAseq libraries were prepared using the TruSeq RNA sample preparation kit (Illumina) according to the protocol. Three multiplexes of 12 libraries and one multiplex of 6 libraries, each with one of the 12 indexed adaptors, were pooled. Each pool was sequenced on one flowcell lane on the HiSeq2000 sequencer (Illumina) in a 101 cycle paired end run. 100 base paired end reads were called with CASAVA v1.8 and output in fastq format. Sequence quality was assessed using FastQC. In house scripts were used to trim and filter poor quality bases and sequence reads. Bases with quality score less than 20 were trimmed from the 3 ' end of reads. Reads with mean quality score less than 20 , or greater than $3 \mathrm{~N}$, or final length less than 50 bases were discarded. Only paired reads were retained for alignment.

\section{Tissue specific expression (TSE) analysis}

Paired RNA reads were aligned to the Ensembl annotation release 75 of bovine genome assembly UMD3.1 using TOPHAT2 [34]. Bam files are available from NCBI Sequence Read Archive and can be found using study accession number SRP042639. Custom scripts were used to assess sequencing performance, library quality and alignment quality. RNA-seqQC [35] was used to perform gene body plots: plot of the mean coverage for expressed transcripts from the 5'-3' end, with the lengths of transcripts normalised to 1-100. The python package HTSeq [36] was used to generate a tissue by gene and a tissue by exon count matrix from all TOPHAT2 alignment files. TSE was analysed using the $\mathrm{R}$ software package edgeR [37] defining a design matrix for which the intercept was the overall mean gene expression. A gene was defined as having TSE if it was significantly $(p<0.01)$ differentially expressed and if its expression level in a tissue was greater than two-fold different to the average expression of that gene across all other tissues. Hierarchical clustering of TSE was performed using the R software packages DESeq [38] and gplots (http://cran.r-project.org/web/packages/gplots/).

To determine the proportion of reads from each gene contributing to the transcriptome in each tissue we calculated and plotted the frequency of the proportion of RNA molecules from each gene $(i)$ as $R N A_{i}=\frac{\text { Count }_{i}+10}{\text { Length }_{i} \times T}$,

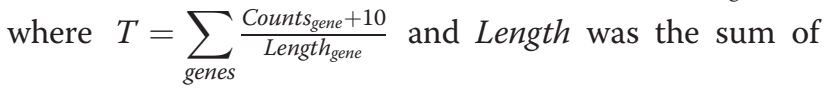
the length of all exons for gene.

To determine where the variation in transcription was occurring the model $y=\mu+$ tissue + replicate + tissue. 
replicate + exon $_{\text {number }}$.tissue + gene + gene. exon + gene. issue was fitted, where $y=\ln \left(1+\right.$ count $\left._{e}\right), \mu$ was mean, tissue was the tissue effect, replicate was the tissue replicate effect, gene was gene effect, exon number was exon effect where exon's are ordered as a fraction of the total exon number and count, was the read count per exon. tissue, replicate, tissue.replicate and

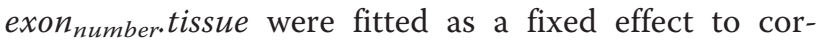
rect for different average coverage per sample (and three samples per replicate). The variance components were then estimated using $\mathrm{R}$.

Functional annotation of the top 200 most significant differentially expressed genes in each tissue was performed using the Database for Annotation, Visualisation and Integrated Discovery (DAVID) v6.7 [39].

\section{Allele specific expression (ASE) analysis}

As a part of the 1000 bull genomes project [10] this cow's whole genome was sequenced to an average fold coverage of 44 (NCBI Sequence Read Archive accession number SRX527665) then SNP and their corresponding phased genotypes were called using an in house pipeline (see [10], for methods). The phased SNP genotypes from Run 3.0 of the 1000 bull genomes project were used in this study to create two parental genomes. For each parental genome, at each SNP position in the UMD3.1 reference, the reference allele was replaced with the allele from the corresponding haplotype. Paired RNA reads were then aligned to each of the parental genomes using TOPHAT2 [34] allowing for two mismatches. Each set of parental alignments were treated individually in the following manner: For each tissue, accepted_hits.bam files from the three replicates were merged, sorted and indexed using SAMtools [40]. Using the list of known heterozygous SNP from the 1000 bull genomes project and using only those that fell within the gene exon boundaries as dictated by Ensembl release 75 annotation of UMD3.1 bovine genome assembly, the SAMtools mpileup function (version 0.1.14) was used to extract allele counts for each SNP. The perl vcfutils.pl varFilter tool was then used with "-Q 0 -a 0 " options to filter the summary VCF file. The 1000 bull genomes pipeline was then also used to perform "private" SNP detection for this cow, using only her whole genome sequence and not any other animals in the 1000 bull genomes project. SNP were then filtered to only consider those that were detected in the "private" variant detection, had a read depth of at least 10 in both parental haplotype alignments and the most abundant allele in the maternal genome was the most abundant allele in paternal genome. This last rule was used to remove regions with obvious mapping bias to the reference genome. SNP were considered as having significant ASE where they had a Chi-squared $\left(\chi^{2}\right)$ value corresponding to $p<0.01$,

$$
X^{2}=\frac{\left(\frac{\left(r_{m} a_{p}-a_{m} r_{p}\right)^{2} N}{\operatorname{ramp}}\right)}{2}
$$

Where $r$ was the count of reference alleles aligned to both the maternal and paternal genomes, $a$ was the count of alternate alleles aligned to both the maternal and paternal genomes, $m$ was the count of reference and alternate alleles aligned to the maternal genome, $p$ was the count of reference and alternate alleles aligned to the paternal genome, $r_{m}$ was the count of reference alleles aligned to the maternal genome, $r_{p}$ was the count of reference alleles aligned to the paternal genome, $a_{m}$ was the count of alternate alleles aligned to the maternal genome, $a_{p}$ was the count of alternate alleles aligned to the paternal genome and $N$ was the total number of alleles aligned to the maternal and paternal genomes. The traditional Chi-squared value was divided by two to account for the value of $N$ being derived from the counts of both parental haplotypes. ASE SNP with a major allele frequency (averaged from both parental genome alignments) greater than or equal to 0.9 were considered to have monoallelic expression (MAE). Because sequence errors would also result in MAE we conducted further filtering of ASE SNP, removing 1) SNP that were not heterozygous in any other animal in the 1000 bull genomes Run3.0 dataset, 2) SNP that fell within regions of the cows genome sequence deemed to be homozygous. Regions of homozygosity were determined by stepping through the genome $50 \mathrm{~kb}$ at a time examining a window of $100 \mathrm{~kb}$ (i.e. $100 \mathrm{~kb}$ windows offset by $50 \mathrm{~kb}$ tiles). The number of heterozygous SNP falling in each window was counted and if less than 6, the region was flagged. If the windows immediately adjacent to flagged regions were also flagged, then the regions were deemed to be homozygous.

Using the sire's genotypes, generated from whole genome sequence, the major allele of all ASE SNP were classified as being either paternally or maternally derived. Not all SNP were able to be classified definitively. These SNP were used to construct a tissue by SNP major allele frequency heat map using the heat map function in the $\mathrm{R}$ software package gplots. To generate this heat map, if a SNP was classified as paternal, then the major allele frequency was unaltered, if the SNP was classified as maternal, then the major allele frequency was multiplied by -1 . An additional tissue by tissue heat map was constructed by transposing a matrix of paternal allele frequencies then multiplying it by the original matrix of paternal allele frequencies. 
To assign a parent-of-origin to genes, a custom $\mathrm{C}++$ program was employed. For each gene, the program generated a ratio of parental classifications from all classified SNP within the gene. If this ratio was greater or equal to 0.75 , the gene was classified as being either maternal or paternal. If the ratio was less than 0.75 , the gene was classified as "undecided". Excluding all "undecided" genes, a Wald-Wolfowitz runs test ( $\mathrm{R}$ software package adehabitat, wawotest function) was performed for each tissue on an ordered list of classified genes to test if the patterns ofexpression, i.e. maternal or paternal, were significantly different from random, identifying regions that could be co-regulated or imprinted.

\section{ASE Validation dataset}

Liver biopsies and blood samples were collected from the 20 first lactation Holstein cows on one occasion. Tissue collection was done with approval from the DEDJTR Animal Ethics Committee (application number 2011-23). Blood was collected by venipuncture of the coccygeal vein and was processed according to the blood fractionation and white blood cell stabilisation procedure in the RiboPure ${ }^{\mathrm{Tm}}$ blood kit (Ambion by Life Technologies) protocol. White blood cells in RNA later were then stored at $-20 \mathrm{oC}$. Liver biopsies were collected by restraining cows in a crush and giving them $10 \mathrm{ml}$ of lignocaine hydrochloride two percent into the subcutaneous, inter-costal and peritoneal tissues at the site of the insertion of the biopsy punch. A small incision was made with a scalpel before a biopsy punch was inserted into the liver to collectapproximately two to three grams of tissue. Following removal of the biopsy punch, betadine cream was placed in the incision site. Cows were given intramuscular antibiotics (Excenel RTU $2 \mathrm{ml} / 100 \mathrm{~kg}$ ) and antiinflammatory drugs (Ketoprofen $2 \mathrm{ml} / 100 \mathrm{~kg}$ ) before being released from the crush. Immediately following collection samples were frozen in liquid nitrogen and then stored at $-80{ }^{\circ} \mathrm{C}$.

Total RNA was extracted from blood samples using the RiboPure ${ }^{\mathrm{Tm}}$ Blood Kit (Ambion) and from liver samples using the RiboPure ${ }^{\mathrm{Ts}}$ Kit (Ambion) as per manufacturer's instructions. RNA quality was assessed on BioAnalyser 2100 (Agilent Technologies). RNAseq libraries were prepared using the TruSeq ${ }^{\mathrm{TM}}$ RNA Sample Preparation Kit v2 (Illumina) according to manufacturer's instructions. Each library was randomly assigned to one of four pools and sequenced on a $\mathrm{HiSeq}^{\mathrm{Tm}} 2000$ (Illumina) in a 105 cycle paired end run. One hundred four base paired end reads were called with CASAVA v1.8 and output in fastq format. Sequence quality was assessed using FastQC. In house scripts were used to trim and filter poor quality bases and sequence reads. Bases with quality score less than 15 were trimmed from the end of reads. Reads with mean quality score less than 20 , or greater than $3 \mathrm{~N}$, or greater three consecutive bases had quality score less than 15 , or final length less than 50 bases were discarded. Only paired reads were retained for alignment.

FImpute [41] was used to impute 28,899,038 SNP utilising the 1000 bull genomes project [10] Run 4.0 phased genotypes as reference population and either real or imputed high density (800 K) SNP genotypes on the individual animals. Where imputed high density SNP genotypes were imputed from BovineSNP50 data. Imputed and phased genotypes for each animal were generated by FImpute and then used to create parental genomes in the same way described above. Paired RNA reads were then aligned to each of the parental genomes using TOPHAT2 [34] with default input parameters.

Similar to the discovery dataset allele counts for each animals predicted heterozygous SNP that fell within annotated coding sequence were calculated and ASE tested using the Chi-squared $\left(\chi^{2}\right)$ test outlined above. Also, a measure of reference allele mapping bias (BIAS) was calculated as

$$
B I A S=\sqrt{\frac{n_{A P_{A}} n_{B P_{B}}}{n_{A P_{B}} n_{B P_{a}}}}
$$

where $n_{A P \mathrm{~A}}$ was the count of $\mathrm{A}$ alleles mapped to the parental genome with $\mathrm{A}$ allele, $n_{B P \mathrm{~B}}$ was the count of $\mathrm{B}$ alleles mapped to the parental genome with $\mathrm{B}$ allele, $n_{A P \mathrm{~B}}$ was the count of A alleles mapped to the parental genome with the $\mathrm{B}$ allele and $n_{B P \mathrm{~A}}$ was the count of $\mathrm{B}$ alleles mapped to the parental genome with A allele. To remove SNP that were likely to be imputation errors, i.e., predicted to be heterozygous when in fact the animal was homozygous for the UMD3.1 reference allele, and also those with significant reference allele mapping bias, SNP were filtered down to those that had reference allele mapping bias of less than two.

\section{Availability of supporting data}

The data set, in the form of bam files, supporting the results of this article is available in the NCBI Sequence Read Archive, study accession number SRP042639.

\section{Additional files}

Additional file 1: Contains supplementary material including Figures S1 - S7, as well as Tables S1, S2, S6 - S9, S11 - S15. (DOCX 7147 kb)

Additional file 2: Contains supplementary material including Tables S3, S4, S5 and S10. (XLSX $7470 \mathrm{~kb}$ )

Competing interests

The authors declare no competing financial or non-financial interests.

Authors' contributions

AJC conceived and designed the discovery experiment, coordinated and participated in sample collection, sample processing and sequencing, 
performed quality control of sequence reads, developed alignment strategy, supervised alignment and data analysis, manuscript preparation. CJVJ performed alignments, data analysis and manuscript preparation. BJH designed the discovery experiment, participated in sample collection, contributed to data analysis, manuscript preparation. MK performed quality control of sequence reads, alignments, data analysis and manuscript preparation. LCM designed the experiment, coordinated and participated in sample collection, manuscript preparation. CAM designed validation experiment, coordinated and participated in sample collection, sample processing and sequencing of validation samples. TN wrote program to prepare parental genomes, assisted with data analysis and preparation of figures, manuscript preparation. MEG designed the discovery and validation experiments, developed statistical tests, supervised the data analysis, manuscript preparation. All authors read and approved the final manuscript.

\section{Acknowledgements}

The authors would like to acknowledge the expertise of Dr Tristan Jubb, Veterinary Consultant with Livestock Health Systems Australia, who performed the animal dissection. Also the efforts of Brett Mason, Kahlil Lawless, Dr Josquin Tibbits, Dr Elizabeth Ross, Jae Greenwood, Greg Morris, Gareth Phillips, Serge Ziero, Alan McDonald and David Clarkson in tissue sample preparation. Also Lakshmi Krishnan and Sami Hakim for RNA extraction and library preparations. Finally the authors would like to acknowledge Dairy Futures CRC for funding the project.

\section{Author details}

${ }^{1}$ Department of Economic Development, Jobs, Transport and Resources, Agribiosciences Building, 5 Ring Rd, Bundoora, Australia. ²Dairy Futures Cooperative Research Centre, Agribiosciences Building, 5 Ring Rd, Bundoora, Australia. ' ${ }^{3}$ a Trobe University, Agribiosciences Building, 5 Ring Rd, Bundoora, Australia. ${ }^{4}$ Department of Economic Development, Jobs, Transport and Resources, 1301 Hazeldean Rd, Ellinbank, Australia. Institute of Land and Food, University of Melbourne, Royal Parade, Parkville, Australia.

\section{Received: 31 July 2015 Accepted: 31 October 2015}

\section{Published online: 23 November 2015}

\section{References}

1. Pai AA, Pritchard JK, Gilad Y. The Genetic and Mechanistic Basis for Variation in Gene Regulation. PLoS Genet. 2015;11:e1004857.

2. Kleinjan DA, van Heyningen V. Long-range control of gene expression: Emerging mechanisms and disruption in disease. Am J Hum Genet. 2005;76:8-32.

3. Wray GA. The evolutionary significance of cis-regulatory mutations. Nat Rev Genet. 2007:8:206-16.

4. Guenther CA, Tasic B, Luo L, Bedell MA, Kingsley DM. A molecular basis for classic blond hair color in Europeans. Nat Genet. 2014;46:748-52.

5. Karim L, Takeda H, Lin L, Druet T, Arias JAC, Baurain D, et al. Variants modulating the expression of a chromosome domain encompassing PLAG1 influence bovine stature. Nat Genet. 2011:43:405-15.

6. Crowley JJ, Zhabotynsky V, Sun W, Huang S, Pakatci IK, Kim Y et al. Analyses of allele-specific gene expression in highly divergent mouse crosses identifies pervasive allelic imbalance. Nat Genet. 2015, Advanced online article.

7. GTEx Consortium. The Genotype-Tissue Expression (GTEx) pilot analysis: Multitissue gene regulation in humans. Science. 2015;348:648-60.

8. Degner JF, Marioni JC, Pai AA, Pickrell JK, Nkadori E, Gilad Y, et al. Effect of read-mapping biases on detecting allele-specific expression from RNA-sequencing data. Bioinformatics. 2009;25:3207-12.

9. Baran Y, Subramaniam M, Biton A, Tukiainen T, Tsang EK, Rivas MA et al. The landscape of genomic imprinting across diverse adult human tissues. Genome Res. 2015, Ahead of print.

10. Daetwyler HD, Capitan A, Pausch H, Stothard P, Van Binsbergen R, Brøndum RF, et al. Whole-genome sequencing of 234 bulls facilitates mapping of monogenic and complex traits in cattle. Nat Genet. 2014;46:858-65.

11. MacLeod IM, Larkin DM, Lewin HA, Hayes BJ, Goddard ME. Inferring demography from runs of homozygosity in whole-genome sequence, with correction for sequence errors. Mol Biol Evol. 2013;30:2209-23.

12. Wang $X$, Clark AG. Using next-generation RNA sequencing to identify imprinted genes. Heredity. 2014;113:156-66.
13. Wood DLA, Nones K, Steptoe A, Christ A, Harliwong I, Newell F, et al. Recommendations for Accurate Resolution of Gene and Isoform Allele-Specific Expression in RNA-Seq Data. PLoS ONE. 2015;10:e0126911.

14. Pant PVK, Tao H, Beilharz EJ, Ballinger DG, Cox DR, Frazer KA. Analysis of allelic differential expression in human white blood cells. Genome Res. 2006;16:331-9.

15. Gao C, Devarajan K, Zhou Y, Slater CM, Daly MB, Chen X. Identifying breast cancer risk loci by global differential allele-specific expression (DASE) analysis in mammary epithelial transcriptome. BMC Genomics. 2012;13:570.

16. Gregg C, Zhang J, Weissbourd B, Luo S, Schroth GP, Haig D, et al. High-resolution analysis of parent-of-origin allelic expression in the mouse brain. Science. 2010;329:643-8.

17. Asselbergs FW, Guo Y, Van Iperen EPA, Sivapalaratnam S, Tragante V, Lanktree MB, et al. Large-scale gene-centric meta-analysis across 32 studies identifies multiple lipid loci. Am J Hum Genet. 2012;91:823-38.

18. Guo T, Yin RX, Chen X, Bin Y, Nie RJ, Li H. Sex-specific association of the SPTY2D1 rs7934205 polymorphism and serum lipid levels. Int J Clin Exp Pathol. 2015;8:665-81.

19. Keane TM, Goodstadt L, Danecek P, White MA, Wong K, Yalcin B, et al. Mouse genomic variation and its effect on phenotypes and gene regulation. Nature. 2011;477:289-94.

20. Pinter SF, Colognori D, Beliveau BJ, Sadreyev RI, Payer B, Yildirim E et al. Allelic imbalance is a prevalent and tissue-specific feature of the mouse transcriptome. Genet. 2015, 200:ahead of print.

21. Römer AM, Lühr I, Klein A, Friedl A, Sebens S, Rösel F, et al. Normal mammary fibroblasts induce reversion of the malignant phenotype in human primary breast cancer. Anticancer Res. 2013;33:1525-36.

22. Erkko H, Pylkäs K, Karppinen SM, Winqvist R. Germline alterations in the CLSPN gene in breast cancer families. Cancer Lett. 2008;261:93-7.

23. Pelden $S$, Insawang T, Thuwajit $C$, Thuwajit $P$. The trefoil factor 1 (TFF1) protein involved in doxorubicin-induced apoptosis resistance is upregulated by estrogen in breast cancer cells. Oncol Rep. 2013;30:1518-26.

24. Itsumura N, Inamo Y, Okazaki F, Teranishi F, Narita H, Kambe T, et al. Compound Heterozygous Mutations in SLC30A2/ZnT2 Results in Low Milk Zinc Concentrations: A Novel Mechanism for Zinc Deficiency in a Breast-Fed Infant. PLOS ONE. 2013;8.

25. Wang W, Lv N, Zhang S, Shui G, Qian H, Zhang J, et al. Cidea is an essential transcriptional coactivator regulating mammary gland secretion of milk lipids. Nat Med. 2012;18:235-43.

26. Vestergaard E, Nexo E, Wendt A, Guthmann F. Trefoil factors in human milk. Early Hum Dev. 2008:84:631-5.

27. Gimelbrant A, Hutchinson JN, Thompson BR, Chess A. Widespread monoallelic expression on human autosomes. Science. 2007;318:1136-40.

28. Zwemer LM, Zak A, Thompson BR, Kirby A, Daly MJ, Chess A, et al. Autosomal monoallelic expression in the mouse. Genome Biol. 2012;13:R10.

29. Borel C, Ferreira PG, Santoni F, Delaneau O, Fort A, Popadin KY, et al. Biased Allelic Expression in Human Primary Fibroblast Single Cells. Am J Hum Genet. 2015;96:70-80.

30. Nitta T, Takahama Y. The lymphocyte guard-IANs: regulation of lymphocyte survival by IAN/GIMAP family proteins. Trends Immunol. 2007;28:58-65.

31. Andersson L, Archibald AL, Bottema CD, Brauning R, Burgess SC, Burt DW, et al. Coordinated international action to accelerate genome-to-phenome with FAANG, the Functional Annotation of Animal Genomes project. Genome Biol. 2015;16:57

32. Cheng HH, Perumbakkam S, Black-Pyrkosz A, Dunn JR, Muir WM. AlleleSpecific Expression Screening Demonstrates that Variation in Genetic Resistance to Marek's Disease in Chicken is Mainly Controlled at the Transcriptional Level. In. 10th World Congress of Genetics Applied to Livestock Production Conference Proceedings. Vancouver, Canada; 2014.

33. Muir WM, Perumbakkam S, Black-Pyrkosz A, Dunn JR, Cheng HH. Allele-Specific Expression, a New Genomics Tool for Development of Value-Added SNP chips and to Fine Map QTL. In. 10th World Congress of Genetics Applied to Livestock Production. Vancouver, Canada; 2014.

34. Kim D, Pertea G, Trapnell C, Pimentel H, Kelley R, Salzberg SL. TopHat2: Accurate alignment of transcriptomes in the presence of insertions, deletions and gene fusions. Genome Biol. 2013;14.

35. Deluca DS, Levin JZ, Sivachenko A, Fennell T, Nazaire MD, Williams C, et al. RNA-SeQC: RNA-seq metrics for quality control and process optimization. Bioinformatics. 2012;28:1530-2. 
36. Anders S, Theodor Pyl P, Huber W. HTSeq - A Python framework to work with high-throughput sequencing data. bioRxiv. 2014, preprint.

37. Robinson MD, McCarthy DJ, Smyth GK. edgeR: a Bioconductor package for differential expression analysis of digital gene expression data. Bioinformatics (Oxford, England). 2010;26:139-40.

38. Anders $\mathrm{S}$, Huber W. Differential expression analysis for sequence count data. Genome Biol. 2010;11.

39. Huang DW, Sherman BT, Lempicki RA. Systematic and integrative analysis of large gene lists using DAVID bioinformatics resources. Nat Protoc. 2009;4:44-57.

40. Li H, Durbin R. Fast and accurate short read alignment with Burrows-Wheeler transform. Bioinformatics. 2009;25:1754-60.

41. Sargolzaei M, Chesnais JP, Schenkel FS. A new approach for efficient genotype imputation using information from relatives. BMC Genomics. 2014;15:478

\section{Submit your next manuscript to BioMed Central and take full advantage of:}

- Convenient online submission

- Thorough peer review

- No space constraints or color figure charges

- Immediate publication on acceptance

- Inclusion in PubMed, CAS, Scopus and Google Scholar

- Research which is freely available for redistribution 